CRYSTALLOGRAPHIC COMMUNICATIONS

ISSN 2056-9890

Received 18 February 2021

Accepted 31 May 2021

Edited by G. Díaz de Delgado, Universidad de Los Andes, Venezuela

Keywords: crystal structure; strontium; carboxylates; N-methyldiethanolamine; coordination polymer; zirconium.

CCDC reference: 2087088

Supporting information: this article has supporting information at journals.iucr.org/e
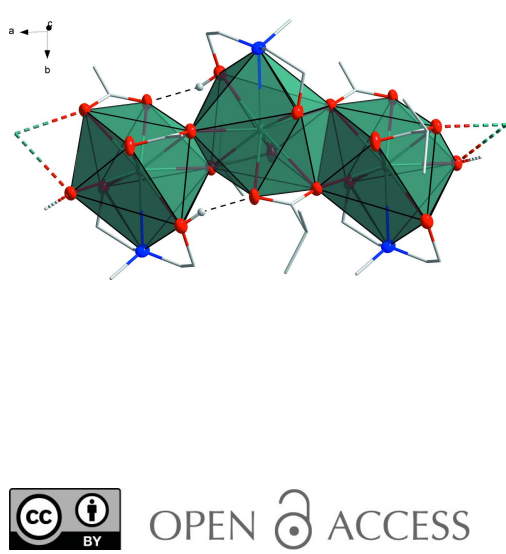

OPEN $\partial$ ACCESS

\section{Synthesis and crystal structure of a one-dimensional chain-like strontium(II) coordination polymer built of $N$-methyldiethanolamine and isobutyrate ligands}

\author{
Maximilian Seiss, Sebastian Schmitz, Martin Börner and Kirill Yu. Monakhov*
}

Leibniz Institute of Surface Engineering (IOM), Permoserstr. 15, 04318 Leipzig, Germany. *Correspondence e-mail: kirill.monakhov@iom-leipzig.de

The one-dimensional coordination polymer (I) $\left[\mathrm{Sr}(\mathrm{ib})_{2}\left(\mathrm{H}_{2} \mathrm{mda}\right)\right]_{n}(\mathrm{Hib}=$ isobutyric acid, $\mathrm{C}_{4} \mathrm{H}_{8} \mathrm{O}_{2}$, and $\mathrm{H}_{2}$ mda $=N$-methyldiethanolamine, $\mathrm{C}_{5} \mathrm{H}_{13} \mathrm{NO}_{2}$ ), namely, catena-poly $\left[\left[\left(N\right.\right.\right.$-methyldiethanolamine- $\left.\kappa^{3} O, N, O^{\prime}\right)$ strontium(II)]-di- $\mu_{2}$ isobutyrato- $\left.\kappa^{3} O, O^{\prime}: O ; \kappa^{3} O: O, O^{\prime}\right]$, was prepared by the one-pot aerobic reaction of $\left[\mathrm{Zr}_{6} \mathrm{O}_{4}(\mathrm{OH})_{4}(\mathrm{ib})_{12}\left(\mathrm{H}_{2} \mathrm{O}\right)\right] \cdot 3 \mathrm{Hib}$ with $\mathrm{Sr}\left(\mathrm{NO}_{3}\right)_{2}$ and $\mathrm{H}_{2}$ mda in the presence of $\mathrm{MnCl}_{2}$ and $\mathrm{Et}_{3} \mathrm{~N}$ in acetonitrile. The use of $\mathrm{MnCl}_{2}$ is key to the isolation of $\mathbf{I}$ as high-quality colorless crystals in good yield. The molecular solid-state structure of I was determined by single-crystal X-ray diffraction. Compound I crystallizes in the monoclinic space group $P 2_{1} / c$ and shows a one-dimensional polymeric chain structure. Each monomeric unit of this coordination polymer consists of a central $\mathrm{Sr}^{\mathrm{II}}$ ion in the $\mathrm{NO}_{8}$ coordination environment of two deprotonated $\mathrm{ib}^{-}$ ligands and one fully protonated $\mathrm{H}_{2}$ mda ligand. The $\mathrm{C}$ and $\mathrm{O}$ atoms of the $\mathrm{H}_{2}$ mda ligand were refined as disordered over two sets of sites with site occupancies of 0.619 (3) and 0.381 (3). Compound I shows thermal stability up to $130^{\circ} \mathrm{C}$ in air.

\section{Chemical context}

Simple metal isobutyrate salts such as $T M(\mathrm{ib})_{2}($ e.g. $T M=\mathrm{Mn}$, $\mathrm{Co}$ and $\mathrm{Ni}$; $\mathrm{Hib}=$ isobutyric acid) and $A M(\mathrm{ib})($ e.g. $A M=\mathrm{Na}$ and $\mathrm{K}$ ) are known to act as precursor materials for the synthesis of a wide variety of polynuclear coordination complexes, e.g. $\left[\mathrm{Mn}^{\mathrm{II}}{ }_{4} \mathrm{Mn}^{\mathrm{III}}{ }_{2}(\mathrm{ib})_{8}(\mathrm{Hbda})_{2}(\mathrm{bda})_{2}\right]\left(\mathrm{H}_{2} \mathrm{bda}=N\right.$-butyldiethanolamine), $\left[\mathrm{Mn}_{4}^{\mathrm{II}} \mathrm{Co}_{2}^{\mathrm{III}}(\mathrm{ib})_{8}(\mathrm{Hmda})_{2}(\mathrm{mda})_{2}\right] \quad$ (Malaestean et al., 2010), $\left[\mathrm{Co}_{3}{ }_{3} \mathrm{Co}_{2}{ }_{2}(\mathrm{Hbda})_{2}(\mathrm{bda})_{2}(\mathrm{ib})_{6}\right] \cdot 2 \mathrm{MeCN}$ and $\left[\mathrm{Ni}_{4}{ }_{4}(\mathrm{Hbda})_{3}(\mathrm{ib})_{5}(\mathrm{MeCN})\right]$ (Schmitz et al., 2016), $\left[\mathrm{Gd}^{\mathrm{III}}{ }_{4} M^{\mathrm{II}}{ }_{8}(\mathrm{OH})_{8}(\mathrm{Lig})_{8}(\mathrm{ib})_{8}\right]\left(\mathrm{ClO}_{4}\right)_{4}\left(M=\mathrm{Zn}^{\mathrm{II}}\right.$ or $\mathrm{Cu}^{\mathrm{II}}, \mathrm{HLig}$ $=2$-(hydroxymethyl)pyridine); Hooper et al., 2012) and $\left[\mathrm{Cr}_{3} \mathrm{O}(\mathrm{ib})_{6}\left(\mathrm{H}_{2} \mathrm{O}\right)_{3}\right]\left(\mathrm{NO}_{3}\right)$ (Parsons et al., 2000). The formation of these polynuclear homo- and heterometallic complexes was enabled by the introduction of flexible amino alcohol ligands into the reaction mixtures (Schmitz et al., 2016; Malaestean et al., 2010). We describe here the first example of a coordination polymer composed of monomeric $\mathrm{Sr}^{\mathrm{II}}$ units that are supported by both isobutyrate and amino alcohol ligands. This makes the synthesized compound $\left[\mathrm{Sr}(\mathrm{ib})_{2}\left(\mathrm{H}_{2} \mathrm{mda}\right)\right]_{n}$ (I) an appealing precursor for reactions with transition metal and lanthanide complexes. In addition, I can find application in solvothermal reactions as it is described, e.g. for the transformation of $\left[\mathrm{Co}_{3}{ }_{3} \mathrm{Co}_{2} \mathrm{II}(\mathrm{Hbda})_{2}(\mathrm{bda})_{2}(\mathrm{ib})_{6}\right]$ to $\left[\mathrm{Co}^{\mathrm{II}}{ }_{10}(\mathrm{OH})_{2}(\mathrm{bda})_{6}(\mathrm{ib})_{6}\right]$ (Schmitz et al., 2018). Herein compound I was isolated as colorless crystals from an aerobic reaction, characterized by infrared (IR) spectroscopy, thermogravimetric analysis (TGA) and single-crystal X-ray diffraction. Compound I 
represents a rare class of alkaline earth metal-isobutyrate complexes with a $1 \mathrm{D}$ polymeric structure $\left(c f .\left\{\left[\mathrm{Mg}(\mathrm{ib})_{2^{-}}\right.\right.\right.$ $\left.\left.\left(\mathrm{H}_{2} \mathrm{O}\right)_{3}\right] \cdot \mathrm{H}_{2} \mathrm{O}\right\}_{n}$ (Malaestean et al., 2013)). Remarkably, $\mathrm{MnCl}_{2}$ is crucial in the synthesis of $\mathbf{I}$ for the formation of high-quality single crystals (in 36\% yield) suitable for X-ray diffraction. When the reaction is carried out without $\mathrm{MnCl}_{2}$, poor quality crystalline material is formed in lower yield within several days. For the syntheses of homometallic coordination complexes it is common to use an additional metal salt, which yields a heterometallic reaction mixture, from which the homometallic complex can be obtained selectively as a solid product (Ako et al., 2007; Liu et al., 2018). In 2007, Ako and co-workers described two heptanuclear iron(III) complexes $\left[\mathrm{Fe}^{\mathrm{III}}{ }_{7} \mathrm{O}_{3}(\text { bda })_{3}(\text { piv })_{9}\left(\mathrm{H}_{2} \mathrm{O}\right)_{3}\right]$ and $\left[\mathrm{Fe}^{\mathrm{III}}{ }_{7} \mathrm{O}_{3}(\text { phda })_{3}(\text { piv })_{9^{-}}\right.$ $\left.\left(\mathrm{H}_{2} \mathrm{O}\right)_{3}\right]\left(\mathrm{H}_{2}\right.$ phda $=N$-phenyldiethanolamine and Hpiv = pivalic acid), which were obtained by the reaction of $\left.\left[\mathrm{Fe}_{3} \mathrm{O} \text { (piv) }\right)_{6}\right]$ piv, nickel(II) acetate tetrahydrate $\left(\mathrm{Ni}(\mathrm{OAc})_{2} \cdot-\right.$ $4 \mathrm{H}_{2} \mathrm{O}$ ) and $\mathrm{H}_{2}$ bda or $\mathrm{H}_{2}$ phda in a molar ratio of 1:1:2 using $\mathrm{MeCN}$ as solvent (Ako et al., 2007). Although $\mathrm{Ni}(\mathrm{OAc})_{2} \cdot 4 \mathrm{H}_{2} \mathrm{O}$ was used in an equimolar ratio with the iron(III) precursor, nickel did not incorporate into the final product. Similar to this, Liu et al. (2018) synthesized a hexanuclear $\left[\mathrm{Zn}_{6}(\mathrm{Lig})_{6}\right.$ $\left.(\mathrm{OOCH})_{6}\right]$ complex $\left(\mathrm{HLig}=4^{\prime}\right.$-(4-carboxyphenyl)-2,2':6, $2^{\prime \prime}$ terpyridine) by the reaction of zinc(II) acetate, $\mathrm{Zn}(\mathrm{OAc})_{2}$, with HLig in the presence of praseodymium(III) nitrate hexahydrate, $\operatorname{Pr}\left(\mathrm{NO}_{3}\right)_{3} \cdot 6 \mathrm{H}_{2} \mathrm{O}$, using a 2:2:1 molar ratio. The reaction was performed solvothermally in DMF and praseodymium did not incorporate into the final $\left[\mathrm{Zn}_{6}(\mathrm{Lig})_{6}(\mathrm{OOCH})_{6}\right]$ complex, which was isolated as a pure product by filtration (Liu et al., 2018). Here $\left[\mathrm{Sr}(\mathrm{ib})_{2}\left(\mathrm{H}_{2} \mathrm{mda}\right)\right]_{n}$ was also isolated as a pure product by filtration, which indicates that the additional metal salts (here $\mathrm{MnCl}_{2}$ ) remain in the mother liquor.

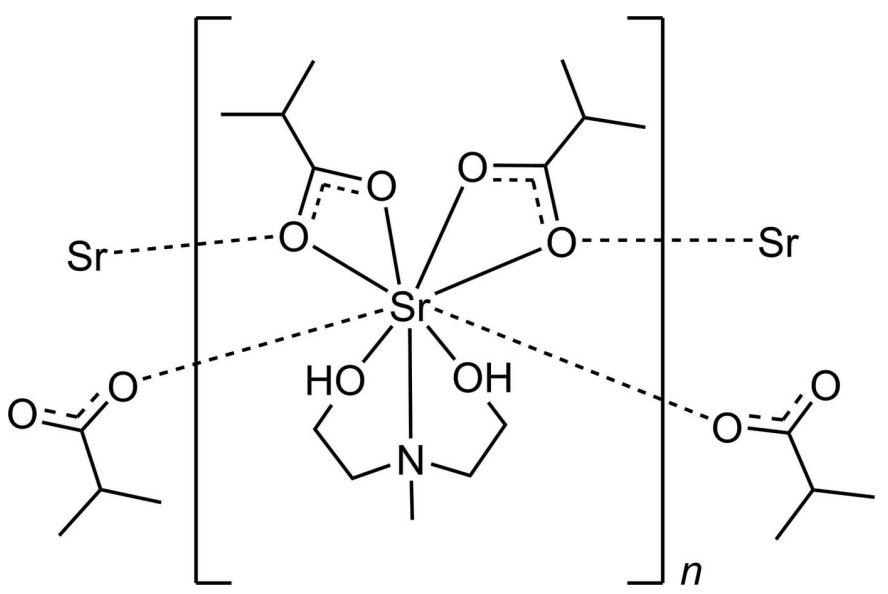

\section{Structural commentary}

The crystal structure consists of a $\mathrm{Sr}^{\mathrm{II}}$ monomer unit (Fig. 1) extending along the $a$-axis direction. The asymmetric unit contains one central $\mathrm{Sr}^{\mathrm{II}}$ ion, which is coordinated by a disordered, tridentate and fully protonated $\mathrm{H}_{2} \mathrm{mda}$ and two deprotonated isobutyrate ligands. In other words, $\mathrm{Sr}^{\mathrm{II}}$ is ninecoordinated by six $\mathrm{O}$ atoms $\left(\mathrm{O} 1, \mathrm{O} 3, \mathrm{O}^{\mathrm{i}}, \mathrm{O}^{\mathrm{ii}}, \mathrm{O}^{\mathrm{i}}\right.$, and $\mathrm{O} 4^{\mathrm{ii}}$;
Table 1

Selected geometric parameters $\left(\AA{ }^{\circ}\right)$.

\begin{tabular}{llll}
\hline $\mathrm{Sr} 1-\mathrm{O} 1^{\mathrm{i}}$ & $2.7563(10)$ & $\mathrm{Sr} 1-\mathrm{O} 5$ & $2.731(11)$ \\
$\mathrm{Sr} 1-\mathrm{O} 3^{\mathrm{ii}}$ & $2.7244(11)$ & $\mathrm{Sr} 1-\mathrm{O} 6$ & $2.66(3)$ \\
$\mathrm{Sr} 1-\mathrm{O} 1$ & $2.5377(10)$ & $\mathrm{Sr} 1-\mathrm{N} 1$ & $2.8495(13)$ \\
$\mathrm{Sr} 1-\mathrm{O} 3$ & $2.5444(10)$ & $\mathrm{Sr} 1 \cdots \mathrm{Sr} 1^{\mathrm{i}}$ & $4.2981(3)$ \\
$\mathrm{Sr} 1-\mathrm{O} 2^{\mathrm{i}}$ & $2.6270(11)$ & $\mathrm{Sr} 1 \cdots \mathrm{Sr} 1^{\mathrm{ii}}$ & $4.2868(3)$ \\
$\mathrm{Sr} 1-\mathrm{O} 4^{\mathrm{ii}}$ & $2.6364(11)$ & &
\end{tabular}

$\mathrm{Sr} 1-\mathrm{O} 1-\mathrm{Sr} 1^{\mathrm{i}} \quad 108.50(4) \quad \mathrm{Sr} 1-\mathrm{O} 1-\mathrm{Sr} 1^{\mathrm{ii}} \quad 108.84(5)$

Symmetry codes: (i) $-x+2,-y+1,-z+1$; (ii) $-x+1,-y+1,-z+1$.

see Table 1 for geometric parameters and symmetry codes) from four different carboxylate groups, two $\mathrm{O}$ atoms (O5 and $\mathrm{O} 6$ or $\mathrm{O} 5 A$ and $\mathrm{O} 6 A$ ) and one $\mathrm{N}$ atom (N1) from the

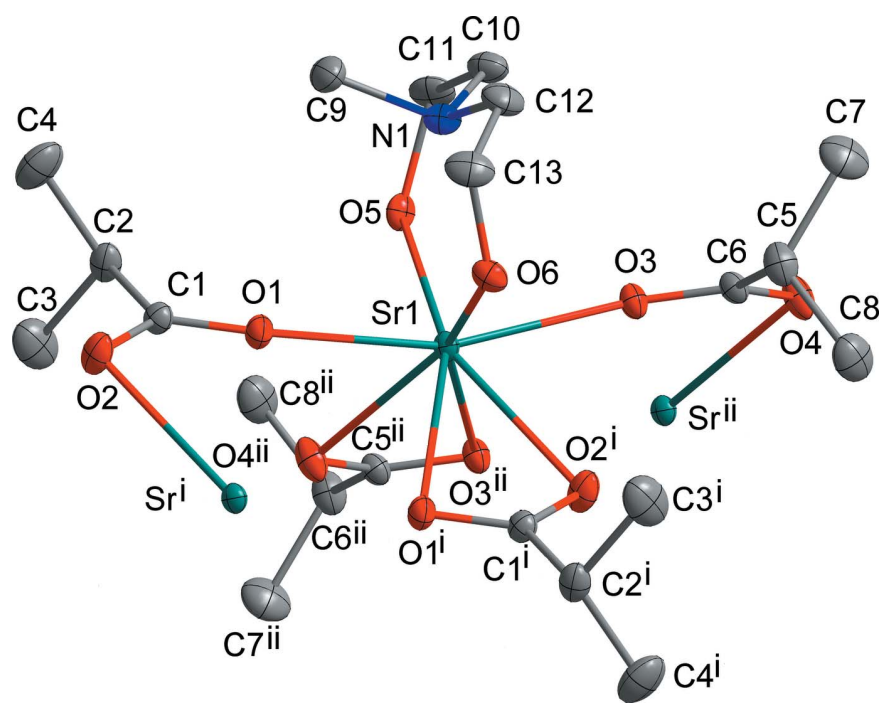

Figure 1

Ellipsoid plot of the monomeric unit of $\mathbf{I}$ with displacement ellipsoids at the $30 \%$ probability level for all non- $\mathrm{H}$ atoms. $\mathrm{H}$ atoms are omitted for clarity. Color code: Sr teal, $\mathrm{C}$ gray, $\mathrm{N}$ blue, $\mathrm{O}$ red. Disordered atoms are omitted for clarity. Symmetry codes: (i) $2-x, 1-y, 1-z$; (ii) $1-x, 1-y$, $1-z$.

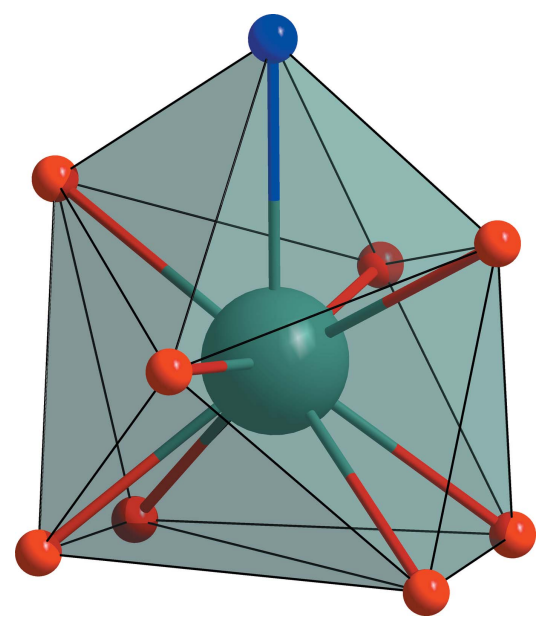

Figure 2

Representation of a polyhedron around a central $\mathrm{Sr}$ ion spanned by the $\mathrm{NO}_{8}$ coordination environment. Color code: $\mathrm{Sr}$ teal, $\mathrm{N}$ blue, O red, polyhedron borders black and polyhedron faces transparent. 
Table 2

Selected Continuous Shapes Measures (CShM) values for the geometry about the nine-coordinate $\mathrm{Sr}^{\mathrm{II}}$ ions of $\mathbf{I}$.

\begin{tabular}{lllll}
\hline Shape & $\begin{array}{l}\text { Capped square } \\
\text { antiprism }\left(C_{4 \mathrm{v}}, \mathrm{J} 10\right)\end{array}$ & $\begin{array}{l}\text { Spherical capped } \\
\text { square antiprism }\left(C_{4 \mathrm{v}}\right)\end{array}$ & $\begin{array}{l}\text { Tricapped trigonal } \\
\text { prism }\left(D_{3 \mathrm{~h}}, \mathrm{~J} 51\right)\end{array}$ & $\begin{array}{l}\text { Spherical tricapped } \\
\text { trigonal prism }\left(D_{3 \mathrm{~h}}\right)\end{array}$ \\
\hline $\mathrm{Sr}^{\mathrm{i}}$ & 4.349 & 3.765 & 5.892 & 3.696 \\
$\mathrm{Sr}^{\mathrm{ii}}$ & 4.026 & 3.346 & 5.575 & 3.423 \\
\hline
\end{tabular}

Symmetry codes: (i) $-x+2,-y+1,-z+1$; (ii) $-x+1,-y+1,-z+1$.

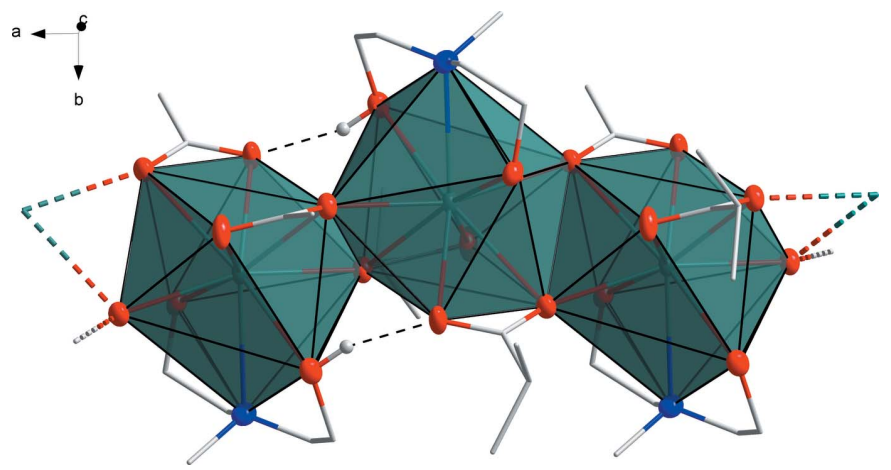

Figure 3

Representation of a segment of the polymeric structure of $\left[\mathrm{Sr}(\mathrm{ib})_{2}\left(\mathrm{H}_{2} \mathrm{mda}\right)\right]_{n}(\mathbf{I})$ along the crystallographic $c$ axis. Color code: $\mathrm{Sr}$ teal, $\mathrm{C}$ gray, $\mathrm{N}$ blue, $\mathrm{O}$ red, bridging $\mathrm{O}$ spheres red and $\mathrm{H}$ atoms white. Hydrogen bonds are shown as dashed black lines. Disordered fragments are omitted for clarity.

$N$-methyldiethanolamine ligand. The resulting coordination environment of the strontium center is $\mathrm{NO}_{8}$. The polyhedral shape of $\mathrm{Sr}$ was evaluated using the $S H A P E$ software version
Table 3

Hydrogen-bond geometry $\left(\AA,^{\circ}\right)$.

\begin{tabular}{lllll}
\hline$D-\mathrm{H} \cdots A$ & $D-\mathrm{H}$ & $\mathrm{H} \cdots A$ & $D \cdots A$ & $D-\mathrm{H} \cdots A$ \\
\hline $\mathrm{O} 6 A-\mathrm{H} 6 A \cdots \mathrm{O} 4^{\mathrm{iii}}$ & 0.84 & 1.83 & $2.61(5)$ & 153 \\
$\mathrm{O} 6-\mathrm{H} 6 B \cdots \mathrm{O} 4^{\mathrm{iii}}$ & 0.84 & 1.95 & $2.75(3)$ & 160 \\
$\mathrm{O} 5-\mathrm{H} 5 \cdots \mathrm{O} 2^{\text {iv }}$ & 0.84 & 1.87 & $2.680(12)$ & 163 \\
\hline
\end{tabular}

Symmetry codes: (iii) $x+1, y, z$; (iv) $x-1, y, z$.

2.1 (Llunell et al., 2013) and can be described as an in-between a distorted spherical capped square antiprism and a distorted spherical tricapped trigonal prism (Fig. 2). The values of the deviation from the ideal geometry are listed in Table 2. The $\mathrm{Sr}-\mathrm{O}_{\mathrm{ib}}$ bond lengths of the bridging $\mathrm{O}$ atoms are between 2.5377 (10) and 2.7563 (10) $\AA$, whereas the non-bridging $\mathrm{Sr}-$ $\mathrm{O}_{\mathrm{ib}}$ bond lengths range from 2.6270 (11) to 2.6364 (11) $\AA$. The non-bonding Sr ‥Sr distances are 4.2869 (3) and 4.2982 (3) A with $\mathrm{Sr}-\mathrm{O}-\mathrm{Sr}$ angles of 108.50 (4) and $108.84(5)^{\circ}$. The $\mathrm{Sr}-$ $\mathrm{O}_{\mathrm{H} 2 \mathrm{mda}}$ bond lengths range between $2.582(20)$ and 2.731 (11) $\AA$, and $\mathrm{Sr}-\mathrm{N}$ bond length is 2.8495 (13) $\AA$.
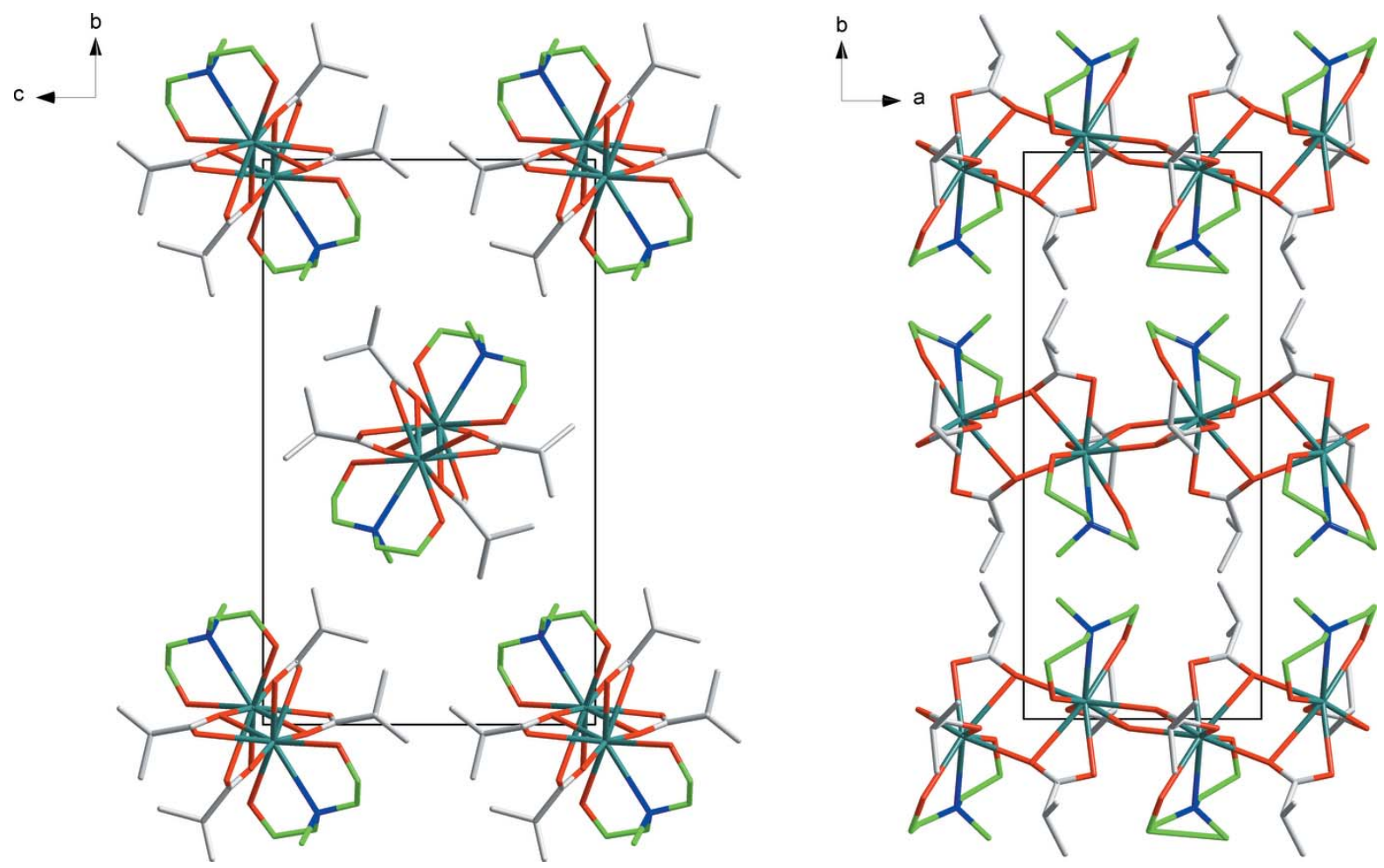

Figure 4

Packing diagram of $\mathbf{I}$, viewed down the $a$ axis (left) and the $c$ axis (right). Color code: Sr teal, $\mathrm{C}$ (ib) gray, $\mathrm{C}$ ( $\left.\mathrm{H}_{2} \mathrm{mda}\right)$ green, $\mathrm{N}$ blue, $\mathrm{O}$ red. $\mathrm{H}$ atoms and disordered fragments are omitted for clarity. 


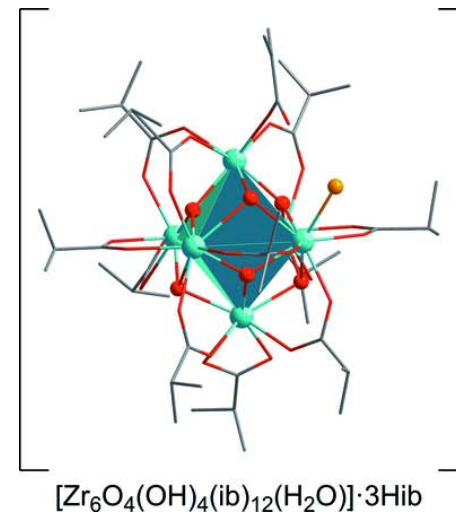

Figure 5

Synthesis of compound $\mathbf{I}$.

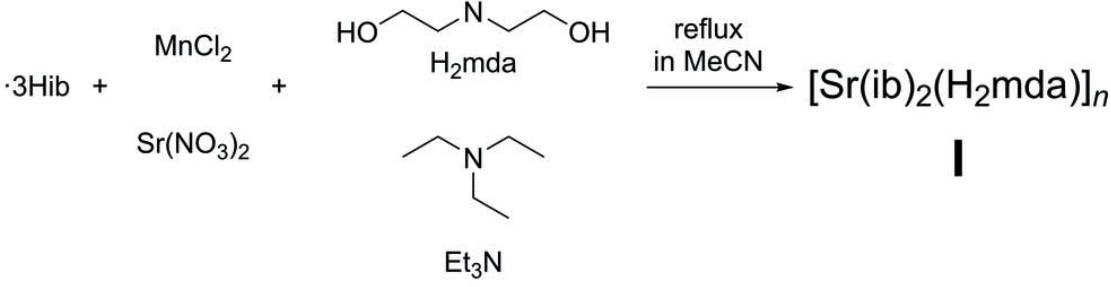

\section{Supramolecular features}

The crystal packing reveals the existence of $1 \mathrm{D}$ polymeric zigzag chains running along the $a$-axis direction (Figs. 3 and 4), in which monomeric $\mathrm{Sr}^{\mathrm{II}}$ units are interlinked by one $\mathrm{O}$ atom of each isobutyrate ligand, which are all coordinated in a chelating, bridging $\mu_{2}-\eta^{2}: \eta^{1}$ mode. The $\mathrm{H}_{2}$ mda ligands coordinate in the chelating $\mu_{1}-\eta^{1}: \eta^{1}: \eta^{1}$ mode to the Sr centers of $\mathbf{I}$. The edge-sharing $\mathrm{SrNO}_{8}$ polyhedra are linked by the isobutyrate $\mathrm{O} 1$ and $\mathrm{O}_{1}^{\mathrm{ii}}$ atoms on the one side and $\mathrm{O} 3$ and $\mathrm{O}^{\mathrm{i}}$ atoms on the other side. Intramolecular hydrogen bonding is present along the chains via $\mathrm{O} 5-\mathrm{H} 5 \cdots \mathrm{O} 2, \mathrm{O} 6-\mathrm{H} 6 \cdots \mathrm{O} 4$ and $\mathrm{O} 6 A-\mathrm{H} 6 A \cdots \mathrm{O} 4$ contacts (Fig. 3, Table 3).

\section{Database survey}

A search of the Cambridge Structural Database (CSD, version 5.42, update of November 2020; Groom et al., 2016) resulted in 34 hits for metal complexes ligated by isobutyrate and $\mathrm{N}$-alkyldiethanolamine. To the best of our knowledge, there are no alkaline earth complexes as well as coordination polymers incorporating both ligands. There are four polymeric structures solely containing group two elements and isobutyrate anions: the magnesium complex catena-poly[[triaqua(isobutyrato)- $\kappa O$ )magnesium]- $\mu$-isobutyrato- $\left.\kappa^{2} O: O^{\prime}\right]$ monohydrate, refcode VIQTOG (Malaestean et al., 2013), catenapoly $\left[\left[\mu\right.\right.$-aqua-diaqua $\left(\mu_{3}-2\right.$-methylpropanoato- $\left.\kappa^{4} O: O, O^{\prime}: O^{\prime}\right)$ calcium] 2-methylpropanoate dihydrate], refcode JUWMEW (Samolová \& Fábry, 2020), as well as the isostructural strontium complex, refcode JUWMIA (Samolová \& Fábry, 2020) and the mixed calcium/strontium complex catena-poly $[[\mu-$ aqua-diaqua $\left(\mu_{3}\right.$-2-methylpropanoato- $\left.\kappa^{4} O: O, O^{\prime}: O^{\prime}\right)$ calcium/ strontium] 2-methylpropanoate dihydrate], refcode JUWMOG (Samolová \& Fábry, 2020).

\section{Synthesis and crystallization}

The one-pot reaction of freshly prepared hexanuclear zirconium complex $\left[\mathrm{Zr}_{6} \mathrm{O}_{4}(\mathrm{OH})_{4}(\mathrm{ib})_{12}\left(\mathrm{H}_{2} \mathrm{O}\right)\right] \cdot 3 \mathrm{Hib}$ (Kogler et al., 2004, abbreviated as $\left.\left\{\mathrm{Zr}_{6}\right\}\right)$ with strontium(II) nitrate and manganese(II) chloride in a 1.0:2.2:2.2, molar ratio was performed in acetonitrile under aerobic conditions, involving 11.1 eq. of $N$-methyldiethanolamine as a co-ligand and 4.0 eq. of triethylamine as a base (see Fig. 5). The polymeric coordination complex $\left[\mathrm{Sr}(\mathrm{ib})_{2}\left(\mathrm{H}_{2} \mathrm{mda}\right)\right]_{n}(\mathbf{I})$ was isolated as colorless crystals. By-products could not be identified. The IR spectrum of $\mathbf{I}$ is characterized by the asymmetric $\mathrm{O}-\mathrm{C}-\mathrm{O}$ vibration bands at $1556 \mathrm{~cm}^{-1}$ and the symmetric $\mathrm{O}-\mathrm{C}-\mathrm{O}$ ones in the range of $1366-1426 \mathrm{~cm}^{-1}$.

The TGA curve (Fig. 6) shows that the thermal decomposition of $\mathbf{I}$ occurs between 130 and $440^{\circ} \mathrm{C}$ with a mass loss of $\mathrm{C}_{12} \mathrm{H}_{27} \mathrm{NO}_{3}$ per monomer unit $\left(\Delta m_{\text {total }}=60.00 \%\right.$ vs $\Delta m_{\text {calcd. }}=$ $61.25 \%$ ), and it yields $\mathrm{SrCO}_{3}$. Overall, the thermal stability of $\mathbf{I}$ up to $130^{\circ} \mathrm{C}$ in air is similar to that determined for isobutyrate diethanolamine complexes of cobalt $\left(140^{\circ} \mathrm{C}\right)$ and nickel $\left(130^{\circ} \mathrm{C}\right)($ Schmitz et al., 2016).

\section{Refinement}

Crystal data, data collection and structure refinement details are summarized in Table 4. The structure was solved using

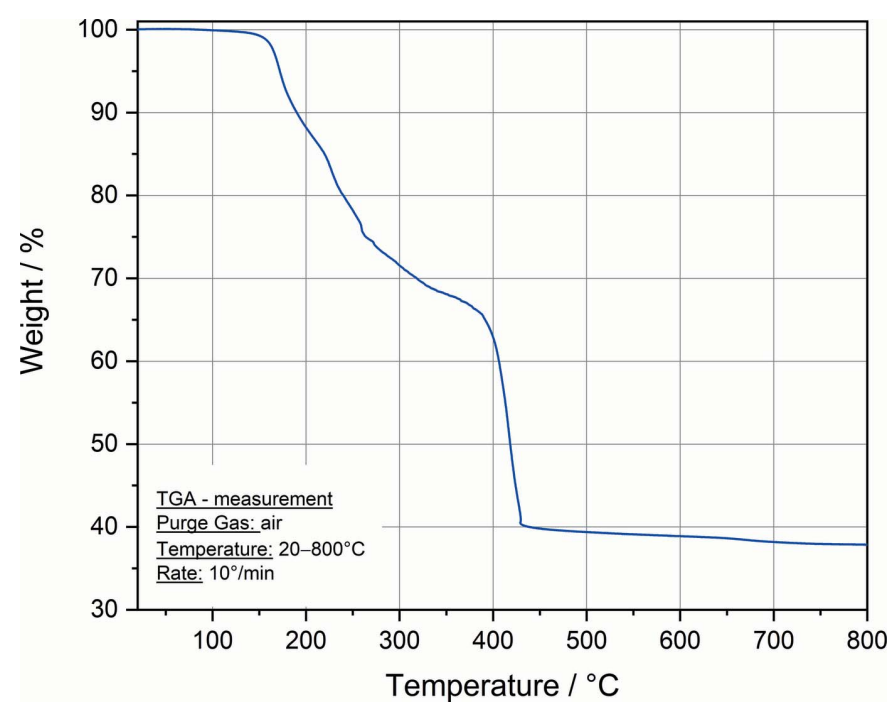

Figure 6

Thermogravimetric analysis for $\mathbf{I}$. 
Table 4

Experimental details.

\begin{tabular}{|c|c|}
\hline \multicolumn{2}{|l|}{ Crystal data } \\
\hline Chemical formula & {$\left[\mathrm{Sr}\left(\mathrm{C}_{4} \mathrm{H}_{7} \mathrm{O}_{2}\right)_{2}\left(\mathrm{C}_{5} \mathrm{H}_{13} \mathrm{NO}_{2}\right)\right]$} \\
\hline$M_{\mathrm{r}}$ & 380.97 \\
\hline Crystal system, space group & Monoclinic, $P 2_{1} / c$ \\
\hline Temperature $(\mathrm{K})$ & 180 \\
\hline$a, b, c(\AA)$ & $8.1516(2), 19.1921(6), 11.4288(3)$ \\
\hline$\beta\left({ }^{\circ}{ }_{0}\right.$ & $99.295(2)$ \\
\hline$V\left(\AA^{6}\right)$ & $1764.52(8)$ \\
\hline$Z$ & 4 \\
\hline Radiation type & $\mathrm{Cu} K \alpha$ \\
\hline$\mu\left(\mathrm{mm}^{-1}\right)$ & 4.46 \\
\hline Crystal size (mm) & $0.28 \times 0.21 \times 0.13$ \\
\hline \multicolumn{2}{|l|}{ Data collection } \\
\hline Diffractometer & Stoe Stadivari \\
\hline Absorption correction & $\begin{array}{l}\text { Multi-scan ( } X \text {-AREA LANA; Stoe, } \\
\text { 2019) }\end{array}$ \\
\hline$T_{\min }, T_{\max }$ & $0.178,0.458$ \\
\hline $\begin{array}{l}\text { No. of measured, independent and } \\
\text { observed }[I>2 \sigma(I)] \text { reflections }\end{array}$ & $15496,3300,3009$ \\
\hline$R_{\text {int }}$ & 0.014 \\
\hline$(\sin \theta / \lambda)_{\max }\left(\AA^{-1}\right)$ & 0.611 \\
\hline \multicolumn{2}{|l|}{ Refinement } \\
\hline$R\left[F^{2}>2 \sigma\left(F^{2}\right)\right], w R\left(F^{2}\right), S$ & $0.018,0.046,1.06$ \\
\hline No. of reflections & 3300 \\
\hline No. of parameters & 240 \\
\hline H-atom treatment & $\mathrm{H}$-atom parameters constrained \\
\hline$\Delta \rho_{\max }, \Delta \rho_{\min }\left(\mathrm{e} \AA^{-3}\right)$ & $0.39,-0.19$ \\
\hline
\end{tabular}

Computer programs: $X$-AREA Pilatus3_SV, Recipe and Integrate (Stoe, 2019), olex2.solve (Bourhis et al., 2015), SHELXL2018/3 (Sheldrick, 2015) and OLEX2 (Dolomanov et al., 2009).

dual space methods and refined by full-matrix least-squares minimization on $F^{2}$. The coordinates of all non-hydrogen atoms were refined with anisotropic thermal parameters. All $\mathrm{H}$ atoms were placed in geometrically idealized positions and refined using a rigid model and included as riding atoms, with methyl $\mathrm{C}-\mathrm{H}=0.98 \AA$, methylene $\mathrm{C}-\mathrm{H}=0.99 \AA$, methine $\mathrm{C}-\mathrm{H}=1.00 \AA$ and $\mathrm{O}-\mathrm{H}=0.84 \AA$. Isotropic displacement parameters were set to $U_{\text {iso }}(\mathrm{H})=1.2 U_{\text {eq }}$ for the parent atom (1.5 for methyl and hydroxy groups). The hydroxy groups and the idealized methyl group were refined as rotating. Atoms C9, $\mathrm{C} 10, \mathrm{C} 11, \mathrm{C} 12, \mathrm{C} 13$, $\mathrm{O} 5$ and $\mathrm{O} 6$ of the $\mathrm{H}_{2}$ mda ligand were refined as disordered over two sets of sites with site occupancies of 0.619 (3) and 0.381 (3). As a result of the short distance between the disordered atoms C11, C13, O5, O6 and their corresponding counterparts, EADP constraints were applied to equalize the displacement ellipsoids of the atom pairs.

\section{Acknowledgements}

The authors are grateful to Professor H. Krautscheid (Leipzig University) for access to the single-crystal X-ray diffraction equipment.

\section{Funding information}

Funding for this research was provided by: the Leibniz Association through the Leibniz Collaborative Excellence funding program (iMolKit).

\section{References}

Ako, A. M., Waldmann, O., Mereacre, V., Klöwer, F., Hewitt, I. J., Anson, C. E., Güdel, H. U. \& Powell, A. K. (2007). Inorg. Chem. 46, 756-766.

Bourhis, L. J., Dolomanov, O. V., Gildea, R. J., Howard, J. A. K. \& Puschmann, H. (2015). Acta Cryst. A71, 59-75.

Dolomanov, O. V., Bourhis, L. J., Gildea, R. J., Howard, J. A. K. \& Puschmann, H. (2009). J. Appl. Cryst. 42, 339-341.

Groom, C. R., Bruno, I. J., Lightfoot, M. P. \& Ward, S. C. (2016). Acta Cryst. B72, 171-179.

Hooper, T. N., Schnack, J., Piligkos, S., Evangelisti, M. \& Brechin, E. K. (2012). Angew. Chem. Int. Ed. 51, 4633-4636.

Kogler, F. R., Jupa, M., Puchberger, M. \& Schubert, U. (2004). J. Mater. Chem. 14, 3133-3138.

Liu, X., Gao, P. \& Hu, M. (2018). Polyhedron, 144, 119-124.

Llunell, M., Casanova, D., Cirera, A., Alemany, A. \& Alvarez, S. (2013). SHAPE. University of Barcelona, Barcelona, Spain.

Malaestean, I. L., Schmitz, S., Ellern, A. \& Kögerler, P. (2013). Acta Cryst. C69, 1144-1146.

Malaestean, I. L., Speldrich, M., Ellern, A., Baca, S. G. \& Kögerler, P. (2010). Polyhedron, 29, 1990-1997.

Parsons, S., Smith, A. A. \& Winpenny, R. E. P. (2000). Chem. Commun. pp. 579-580.

Samolová, E. \& Fábry, J. (2020). Acta Cryst. E76, 1684-1688.

Schmitz, S., Monakhov, K. Yu., van Leusen, J., Izarova, N. V., Hess, V. \& Kögerler, P. (2016). RSC Adv. 6, 100664-100669.

Schmitz, S., Secker, T., Batool, M., van Leusen, J., Nadeem, M. A. \& Kögerler, P. (2018). Inorg. Chim. Acta, 482, 522-525.

Sheldrick, G. M. (2015). Acta Cryst. C71, 3-8.

Stoe (2019). $X$-AREA. Stoe \& Cie, Darmstadt, Germany. 


\section{supporting information}

Acta Cryst. (2021). E77, 703-707 [https://doi.org/10.1107/S2056989021005594]

Synthesis and crystal structure of a one-dimensional chain-like strontium(II) coordination polymer built of $N$-methyldiethanolamine and isobutyrate ligands

Maximilian Seiss, Sebastian Schmitz, Martin Börner and Kirill Yu. Monakhov

Computing details

Data collection: $X$-AREA Pilatus3_SV (Stoe, 2019); cell refinement: $X$-AREA Recipe (Stoe, 2019); data reduction: $X$ AREA Integrate (Stoe, 2019); program(s) used to solve structure: olex2.solve (Bourhis et al., 2015); program(s) used to refine structure: SHELXL2018/3 (Sheldrick, 2015); molecular graphics: Olex2 1.3 (Dolomanov et al., 2009); software used to prepare material for publication: X-AREA (Stoe, 2019).

catena-Poly [[( $N$-methyldiethanolamine- $\left.\kappa^{3} O, N, O^{\prime}\right)$ strontium(II)]-di- $\mu_{2}$-isobutyrato- $\left.\kappa^{3} O, O^{\prime}: O ; \kappa^{3} O: O, O \prime\right]$

Crystal data

$\left[\mathrm{Sr}\left(\mathrm{C}_{4} \mathrm{H}_{7} \mathrm{O}_{2}\right)_{2}\left(\mathrm{C}_{5} \mathrm{H}_{13} \mathrm{NO}_{2}\right)\right]$

$M_{r}=380.97$

Monoclinic, $P 2_{1} / c$

$a=8.1516(2) \AA$

$b=19.1921(6) \AA$

$c=11.4288(3) \AA$

$\beta=99.295(2)^{\circ}$

$V=1764.52(8) \AA^{3}$

$Z=4$

Data collection

Stoe Stadivari diffractometer

Radiation source: GeniX 3D HF Cu Graded multilayer mirror monochromator Detector resolution: 5.81 pixels $\mathrm{mm}^{-1}$ rotation method, $\omega$ scans Absorption correction: multi-scan (XAREA LANA; Stoe, 2019)

$T_{\min }=0.178, T_{\max }=0.458$

\section{Refinement}

Refinement on $F^{2}$

Least-squares matrix: full

$R\left[F^{2}>2 \sigma\left(F^{2}\right)\right]=0.018$

$w R\left(F^{2}\right)=0.046$

$S=1.06$

3300 reflections

240 parameters

0 restraints

Primary atom site location: iterative
$F(000)=792$

$D_{\mathrm{x}}=1.434 \mathrm{Mg} \mathrm{m}^{-3}$

$\mathrm{Cu} K \alpha$ radiation, $\lambda=1.54186 \AA$

Cell parameters from 16191 reflections

$\theta=4.5-70.9^{\circ}$

$\mu=4.46 \mathrm{~mm}^{-1}$

$T=180 \mathrm{~K}$

Block, light yellow

$0.28 \times 0.21 \times 0.13 \mathrm{~mm}$

15496 measured reflections

3300 independent reflections

3009 reflections with $I>2 \sigma(I)$

$R_{\text {int }}=0.014$

$\theta_{\max }=70.5^{\circ}, \theta_{\min }=4.6^{\circ}$

$h=-6 \rightarrow 9$

$k=-23 \rightarrow 22$

$l=-13 \rightarrow 13$

Hydrogen site location: inferred from neighbouring sites

$\mathrm{H}$-atom parameters constrained

$w=1 /\left[\sigma^{2}\left(F_{\mathrm{o}}^{2}\right)+(0.031 P)^{2}+0.1664 P\right]$

where $P=\left(F_{\mathrm{o}}^{2}+2 F_{\mathrm{c}}^{2}\right) / 3$

$(\Delta / \sigma)_{\max }=0.002$

$\Delta \rho_{\max }=0.39$ e $\AA^{-3}$

$\Delta \rho_{\min }=-0.19 \mathrm{e} \AA^{-3}$ 


\section{Special details}

Geometry. All esds (except the esd in the dihedral angle between two 1.s. planes) are estimated using the full covariance matrix. The cell esds are taken into account individually in the estimation of esds in distances, angles and torsion angles; correlations between esds in cell parameters are only used when they are defined by crystal symmetry. An approximate (isotropic) treatment of cell esds is used for estimating esds involving l.s. planes.

Fractional atomic coordinates and isotropic or equivalent isotropic displacement parameters $\left(\AA^{2}\right)$

\begin{tabular}{|c|c|c|c|c|c|}
\hline & $x$ & $y$ & $z$ & $U_{\text {iso }} * / U_{\text {eq }}$ & Occ. $(<1)$ \\
\hline Sr1 & $0.74277(2)$ & $0.53023(2)$ & $0.46969(2)$ & $0.02003(5)$ & \\
\hline $\mathrm{O} 1$ & $1.03404(12)$ & $0.57713(5)$ & $0.53587(9)$ & $0.0276(2)$ & \\
\hline N1 & $0.71783(17)$ & $0.65620(7)$ & $0.33604(12)$ & $0.0315(3)$ & \\
\hline $\mathrm{C} 1$ & $1.14299(18)$ & $0.61161(8)$ & $0.60142(13)$ & $0.0248(3)$ & \\
\hline $\mathrm{O} 2$ & $1.29545(13)$ & $0.59817(6)$ & $0.60970(11)$ & $0.0350(3)$ & \\
\hline $\mathrm{C} 2$ & $1.0907(2)$ & $0.66959(9)$ & $0.67912(16)$ & $0.0333(4)$ & \\
\hline $\mathrm{H} 2$ & 0.967393 & 0.675584 & 0.660022 & $0.040^{*}$ & \\
\hline $\mathrm{O} 3$ & $0.44290(13)$ & $0.51634(6)$ & $0.36832(9)$ & $0.0277(2)$ & \\
\hline $\mathrm{C} 3$ & $1.1366(3)$ & $0.64916(12)$ & $0.80933(18)$ & $0.0564(6)$ & \\
\hline $\mathrm{H} 3 \mathrm{~A}$ & 1.079880 & 0.605721 & 0.823650 & $0.085^{*}$ & \\
\hline $\mathrm{H} 3 \mathrm{~B}$ & 1.102401 & 0.686203 & 0.859261 & $0.085^{*}$ & \\
\hline $\mathrm{H} 3 \mathrm{C}$ & 1.257147 & 0.642439 & 0.828695 & $0.085^{*}$ & \\
\hline $\mathrm{O} 4$ & $0.18685(13)$ & $0.48389(7)$ & $0.29816(10)$ & $0.0360(3)$ & \\
\hline $\mathrm{C} 4$ & $1.1738(3)$ & $0.73767(10)$ & $0.6536(2)$ & $0.0579(6)$ & \\
\hline $\mathrm{H} 4 \mathrm{~A}$ & 1.294585 & 0.731263 & 0.665716 & $0.087^{*}$ & \\
\hline $\mathrm{H} 4 \mathrm{~B}$ & 1.145448 & 0.773973 & 0.707215 & $0.087^{*}$ & \\
\hline $\mathrm{H} 4 \mathrm{C}$ & 1.134732 & 0.751626 & 0.571288 & $0.087 *$ & \\
\hline O5A & $0.577(3)$ & $0.6350(9)$ & $0.5311(11)$ & $0.0265(9)$ & $0.381(3)$ \\
\hline $\mathrm{H} 5 \mathrm{~A}$ & 0.609072 & 0.636661 & 0.604661 & $0.040^{*}$ & $0.381(3)$ \\
\hline $\mathrm{C} 5$ & $0.33403(18)$ & $0.49642(8)$ & $0.28442(13)$ & $0.0248(3)$ & \\
\hline O6A & $0.882(6)$ & $0.530(3)$ & $0.273(5)$ & $0.0294(17)$ & $0.381(3)$ \\
\hline H6A & 0.964326 & 0.504679 & 0.267529 & $0.044^{*}$ & $0.381(3)$ \\
\hline C6 & $0.3829(2)$ & $0.48362(10)$ & $0.16298(15)$ & $0.0350(4)$ & \\
\hline H6 & 0.502060 & 0.497040 & 0.166344 & $0.042 *$ & \\
\hline $\mathrm{C} 7$ & $0.2768(3)$ & $0.52822(12)$ & $0.06894(18)$ & $0.0542(6)$ & \\
\hline $\mathrm{H} 7 \mathrm{~A}$ & 0.159415 & 0.515843 & 0.065063 & $0.081^{*}$ & \\
\hline H7B & 0.310497 & 0.519856 & -0.008377 & $0.081^{*}$ & \\
\hline $\mathrm{H} 7 \mathrm{C}$ & 0.292495 & 0.577568 & 0.089822 & $0.081^{*}$ & \\
\hline $\mathrm{C} 8$ & $0.3644(3)$ & $0.40680(11)$ & $0.13232(17)$ & $0.0484(5)$ & \\
\hline $\mathrm{H} 8 \mathrm{~A}$ & 0.432387 & 0.379290 & 0.194290 & $0.073^{*}$ & \\
\hline $\mathrm{H} 8 \mathrm{~B}$ & 0.401459 & 0.398322 & 0.056146 & $0.073^{*}$ & \\
\hline $\mathrm{H} 8 \mathrm{C}$ & 0.247570 & 0.393170 & 0.126731 & $0.073^{*}$ & \\
\hline C9A & $0.5616(6)$ & $0.6538(3)$ & $0.2356(5)$ & $0.0484(14)$ & $0.381(3)$ \\
\hline H9AA & 0.462861 & 0.641832 & 0.270231 & $0.073^{*}$ & $0.381(3)$ \\
\hline H9AB & 0.578748 & 0.618626 & 0.176647 & $0.073 *$ & $0.381(3)$ \\
\hline H9AC & 0.545747 & 0.699558 & 0.197278 & $0.073^{*}$ & $0.381(3)$ \\
\hline $\mathrm{C} 10 \mathrm{~A}$ & $0.6919(7)$ & $0.7102(2)$ & $0.4143(5)$ & $0.0447(13)$ & $0.381(3)$ \\
\hline H10A & 0.658994 & 0.752626 & 0.367158 & $0.054 *$ & $0.381(3)$ \\
\hline H10B & 0.798802 & 0.720209 & 0.466068 & $0.054 *$ & $0.381(3)$ \\
\hline
\end{tabular}




$\begin{array}{llllll}\text { C11A } & 0.5675(15) & 0.6966(8) & 0.4890(14) & 0.0384(12) & 0.381(3) \\ \text { H11A } & 0.581608 & 0.730392 & 0.555314 & 0.046^{*} & 0.381(3) \\ \text { H11B } & 0.455628 & 0.703709 & 0.442146 & 0.046^{*} & 0.381(3) \\ \text { C12A } & 0.8593(6) & 0.6604(2) & 0.2824(4) & 0.0372(12) & 0.381(3) \\ \text { H12A } & 0.957084 & 0.667164 & 0.345023 & 0.045^{*} & 0.381(3) \\ \text { H12B } & 0.849687 & 0.702121 & 0.230877 & 0.045^{*} & 0.381(3) \\ \text { C13A } & 0.893(4) & 0.5941(18) & 0.205(3) & 0.0431(8) & 0.381(3) \\ \text { H13A } & 0.809510 & 0.592742 & 0.131210 & 0.052^{*} & 0.381(3) \\ \text { H13B } & 1.004460 & 0.597745 & 0.181722 & 0.052^{*} & 0.381(3) \\ \text { O6 } & 0.866(3) & 0.5342(16) & 0.268(3) & 0.0294(17) & 0.619(3) \\ \text { H6B } & 0.960128 & 0.517184 & 0.294366 & 0.044^{*} & 0.619(3) \\ \text { O5 } & 0.5875(15) & 0.6406(5) & 0.5558(6) & 0.0265(9) & 0.619(3) \\ \text { H5 } & 0.505903 & 0.619209 & 0.574990 & 0.040^{*} & 0.619(3) \\ \text { C9 } & 0.8519(4) & 0.70964(15) & 0.3830(3) & 0.0411(7) & 0.619(3) \\ \text { H9A } & 0.961638 & 0.690803 & 0.376032 & 0.062^{*} & 0.619(3) \\ \text { H9B } & 0.846963 & 0.719638 & 0.466490 & 0.062^{*} & 0.619(3) \\ \text { H9C } & 0.832910 & 0.752678 & 0.336686 & 0.062^{*} & 0.619(3) \\ \text { C11 } & 0.5250(7) & 0.6997(5) & 0.4694(8) & 0.0384(12) & 0.619(3) \\ \text { H11C } & 0.403861 & 0.705598 & 0.467052 & 0.046^{*} & 0.619(3) \\ \text { H11D } & 0.580042 & 0.743736 & 0.498683 & 0.046^{*} & 0.619(3) \\ \text { C12 } & 0.7389(4) & 0.63928(16) & 0.2165(2) & 0.0404(8) & 0.619(3) \\ \text { H12C } & 0.747000 & 0.683090 & 0.172108 & 0.048^{*} & 0.619(3) \\ \text { H12D } & 0.639044 & 0.613961 & 0.177495 & 0.048^{*} & 0.619(3) \\ \text { C13 } & 0.887(2) & 0.5965(11) & 0.2096(17) & 0.0431(8) & 0.619(3) \\ \text { H13C } & 0.896781 & 0.587366 & 0.125768 & 0.052^{*} & 0.619(3) \\ \text { H13D } & 0.988442 & 0.620832 & 0.247982 & 0.052^{*} & 0.619(3) \\ \text { C10 } & 0.5591(3) & 0.68558(14) & 0.3441(3) & 0.0372(7) & 0.619(3) \\ \text { H10C } & 0.472220 & 0.653633 & 0.304369 & 0.045^{*} & 0.619(3) \\ \text { H10D } & 0.548179 & 0.730028 & 0.299556 & 0.045^{*} & 0.619(3)\end{array}$

Atomic displacement parameters $\left(\AA^{2}\right)$

\begin{tabular}{lllllll}
\hline & $U^{11}$ & $U^{22}$ & $U^{33}$ & $U^{12}$ & $U^{13}$ & $U^{23}$ \\
\hline Sr1 & $0.01411(8)$ & $0.02466(8)$ & $0.02156(8)$ & $-0.00039(4)$ & $0.00361(5)$ & $-0.00108(5)$ \\
O1 & $0.0218(5)$ & $0.0283(5)$ & $0.0319(6)$ & $-0.0029(4)$ & $0.0016(4)$ & $-0.0046(4)$ \\
N1 & $0.0321(8)$ & $0.0296(7)$ & $0.0338(7)$ & $0.0040(5)$ & $0.0082(6)$ & $0.0048(6)$ \\
C1 & $0.0201(7)$ & $0.0262(7)$ & $0.0287(7)$ & $-0.0014(5)$ & $0.0059(5)$ & $-0.0009(6)$ \\
O2 & $0.0176(6)$ & $0.0348(6)$ & $0.0537(7)$ & $-0.0017(4)$ & $0.0092(5)$ & $-0.0128(5)$ \\
C2 & $0.0233(8)$ & $0.0343(8)$ & $0.0418(9)$ & $0.0036(6)$ & $0.0040(6)$ & $-0.0114(7)$ \\
O3 & $0.0203(5)$ & $0.0360(6)$ & $0.0259(5)$ & $-0.0021(4)$ & $0.0010(4)$ & $-0.0006(4)$ \\
C3 & $0.0662(14)$ & $0.0652(14)$ & $0.0378(11)$ & $0.0156(11)$ & $0.0084(9)$ & $-0.0165(10)$ \\
O4 & $0.0177(6)$ & $0.0627(8)$ & $0.0276(6)$ & $-0.0007(5)$ & $0.0043(4)$ & $0.0012(5)$ \\
C4 & $0.0619(14)$ & $0.0340(10)$ & $0.0818(16)$ & $-0.0043(9)$ & $0.0239(11)$ & $-0.0198(10)$ \\
O5A & $0.0273(17)$ & $0.0322(16)$ & $0.021(3)$ & $-0.0033(11)$ & $0.008(3)$ & $-0.0085(19)$ \\
C5 & $0.0200(8)$ & $0.0308(8)$ & $0.0235(7)$ & $0.0022(6)$ & $0.0031(5)$ & $0.0009(6)$ \\
O6A & $0.023(4)$ & $0.035(3)$ & $0.031(2)$ & $0.007(3)$ & $0.007(3)$ & $0.0037(18)$ \\
C6 & $0.0265(9)$ & $0.0539(10)$ & $0.0260(8)$ & $-0.0021(7)$ & $0.0088(6)$ & $-0.0036(7)$ \\
C7 & $0.0684(15)$ & $0.0683(14)$ & $0.0276(9)$ & $0.0088(11)$ & $0.0131(9)$ & $0.0082(9)$
\end{tabular}




\begin{tabular}{lllllll} 
C8 & $0.0536(12)$ & $0.0571(12)$ & $0.0346(10)$ & $0.0073(9)$ & $0.0073(8)$ & $-0.0139(9)$ \\
C9A & $0.037(3)$ & $0.052(3)$ & $0.052(3)$ & $-0.001(2)$ & $-0.005(2)$ & $0.017(2)$ \\
C10A & $0.057(3)$ & $0.026(2)$ & $0.056(3)$ & $-0.003(2)$ & $0.022(2)$ & $-0.002(2)$ \\
C11A & $0.030(3)$ & $0.0315(12)$ & $0.056(3)$ & $0.017(2)$ & $0.015(3)$ & $0.0070(18)$ \\
C12A & $0.039(3)$ & $0.034(2)$ & $0.042(3)$ & $0.0017(18)$ & $0.015(2)$ & $0.015(2)$ \\
C13A & $0.0521(15)$ & $0.0471(17)$ & $0.0352(18)$ & $0.0102(11)$ & $0.0227(11)$ & $0.0109(12)$ \\
O6 & $0.023(4)$ & $0.035(3)$ & $0.031(2)$ & $0.007(3)$ & $0.007(3)$ & $0.0037(18)$ \\
O5 & $0.0273(17)$ & $0.0322(16)$ & $0.021(3)$ & $-0.0033(11)$ & $0.008(3)$ & $-0.0085(19)$ \\
C9 & $0.0362(16)$ & $0.0362(15)$ & $0.0495(18)$ & $-0.0081(12)$ & $0.0024(12)$ & $0.0055(13)$ \\
C11 & $0.030(3)$ & $0.0315(12)$ & $0.056(3)$ & $0.017(2)$ & $0.015(3)$ & $0.0070(18)$ \\
C12 & $0.051(2)$ & $0.0420(16)$ & $0.0275(14)$ & $0.0089(13)$ & $0.0056(12)$ & $0.0089(12)$ \\
C13 & $0.0521(15)$ & $0.0471(17)$ & $0.0352(18)$ & $0.0102(11)$ & $0.0227(11)$ & $0.0109(12)$ \\
C10 & $0.0311(15)$ & $0.0330(14)$ & $0.0478(17)$ & $0.0068(11)$ & $0.0076(12)$ & $0.0106(12)$ \\
\hline
\end{tabular}

Geometric parameters $\left(A,{ }^{\circ}\right)$

\begin{tabular}{|c|c|c|c|}
\hline $\mathrm{Sr} 1-\mathrm{O}^{\mathrm{i}}$ & $2.7563(10)$ & $\mathrm{C} 6-\mathrm{H} 6$ & 1.0000 \\
\hline $\mathrm{Sr} 1-\mathrm{O} 3^{\mathrm{ii}}$ & $2.7244(11)$ & $\mathrm{C} 6-\mathrm{C} 7$ & $1.528(3)$ \\
\hline $\mathrm{Sr} 1-\mathrm{O} 1$ & $2.5377(10)$ & $\mathrm{C} 6-\mathrm{C} 8$ & $1.517(3)$ \\
\hline $\mathrm{Sr} 1-\mathrm{O} 3$ & $2.5444(10)$ & $\mathrm{C} 7-\mathrm{H} 7 \mathrm{~A}$ & 0.9800 \\
\hline $\mathrm{Sr} 1-\mathrm{O} 2^{\mathrm{i}}$ & $2.6270(11)$ & $\mathrm{C} 7-\mathrm{H} 7 \mathrm{~B}$ & 0.9800 \\
\hline $\mathrm{Sr} 1-\mathrm{O} 4^{\mathrm{ii}}$ & $2.6364(11)$ & $\mathrm{C} 7-\mathrm{H} 7 \mathrm{C}$ & 0.9800 \\
\hline $\mathrm{Sr} 1-\mathrm{O} 5$ & $2.731(11)$ & $\mathrm{C} 8-\mathrm{H} 8 \mathrm{~A}$ & 0.9800 \\
\hline $\mathrm{Sr} 1-\mathrm{O} 6$ & $2.66(3)$ & $\mathrm{C} 8-\mathrm{H} 8 \mathrm{~B}$ & 0.9800 \\
\hline $\mathrm{Sr} 1-\mathrm{N} 1$ & $2.8495(13)$ & $\mathrm{C} 8-\mathrm{H} 8 \mathrm{C}$ & 0.9800 \\
\hline $\mathrm{Sr} 1-\mathrm{Sr} 1^{\mathrm{i}}$ & $4.2981(3)$ & C9A-H9AA & 0.9800 \\
\hline $\mathrm{Sr} 1-\mathrm{Sr} 1^{\mathrm{ii}}$ & $4.2868(3)$ & $\mathrm{C} 9 \mathrm{~A}-\mathrm{H} 9 \mathrm{AB}$ & 0.9800 \\
\hline $\mathrm{Sr} 1-\mathrm{O} 5 \mathrm{~A}$ & $2.58(2)$ & $\mathrm{C} 9 \mathrm{~A}-\mathrm{H} 9 \mathrm{AC}$ & 0.9800 \\
\hline $\mathrm{Sr} 1-\mathrm{C} 5^{\mathrm{ii}}$ & $3.0199(15)$ & $\mathrm{C} 10 \mathrm{~A}-\mathrm{H} 10 \mathrm{~A}$ & 0.9900 \\
\hline $\mathrm{Sr} 1-\mathrm{O} 6 \mathrm{~A}$ & $2.68(6)$ & $\mathrm{C} 10 \mathrm{~A}-\mathrm{H} 10 \mathrm{~B}$ & 0.9900 \\
\hline $\mathrm{O} 1-\mathrm{C} 1$ & $1.2541(18)$ & $\mathrm{C} 10 \mathrm{~A}-\mathrm{C} 11 \mathrm{~A}$ & $1.450(16)$ \\
\hline $\mathrm{N} 1-\mathrm{C} 9 \mathrm{~A}$ & $1.571(5)$ & $\mathrm{C} 11 \mathrm{~A}-\mathrm{H} 11 \mathrm{~A}$ & 0.9900 \\
\hline $\mathrm{N} 1-\mathrm{C} 10 \mathrm{~A}$ & $1.407(5)$ & $\mathrm{C} 11 \mathrm{~A}-\mathrm{H} 11 \mathrm{~B}$ & 0.9900 \\
\hline $\mathrm{N} 1-\mathrm{C} 12 \mathrm{~A}$ & $1.393(5)$ & $\mathrm{C} 12 \mathrm{~A}-\mathrm{H} 12 \mathrm{~A}$ & 0.9900 \\
\hline $\mathrm{N} 1-\mathrm{C} 9$ & $1.531(3)$ & $\mathrm{C} 12 \mathrm{~A}-\mathrm{H} 12 \mathrm{~B}$ & 0.9900 \\
\hline $\mathrm{N} 1-\mathrm{C} 12$ & $1.441(3)$ & $\mathrm{C} 12 \mathrm{~A}-\mathrm{C} 13 \mathrm{~A}$ & $1.60(3)$ \\
\hline $\mathrm{N} 1-\mathrm{C} 10$ & $1.428(3)$ & $\mathrm{C} 13 \mathrm{~A}-\mathrm{H} 13 \mathrm{~A}$ & 0.9900 \\
\hline $\mathrm{C} 1-\mathrm{O} 2$ & $1.2578(19)$ & $\mathrm{C} 13 \mathrm{~A}-\mathrm{H} 13 \mathrm{~B}$ & 0.9900 \\
\hline $\mathrm{C} 1-\mathrm{C} 2$ & $1.527(2)$ & O6- - $66 \mathrm{~B}$ & 0.8400 \\
\hline $\mathrm{C} 2-\mathrm{H} 2$ & 1.0000 & $\mathrm{O} 6-\mathrm{C} 13$ & $1.39(4)$ \\
\hline $\mathrm{C} 2-\mathrm{C} 3$ & $1.526(3)$ & $\mathrm{O} 5-\mathrm{H} 5$ & 0.8400 \\
\hline $\mathrm{C} 2-\mathrm{C} 4$ & $1.521(3)$ & $\mathrm{O} 5-\mathrm{C} 11$ & $1.537(12)$ \\
\hline $\mathrm{O} 3-\mathrm{C} 5$ & $1.2563(18)$ & C9- $\mathrm{H} 9 \mathrm{~A}$ & 0.9800 \\
\hline $\mathrm{C} 3-\mathrm{H} 3 \mathrm{~A}$ & 0.9800 & С9-Н9B & 0.9800 \\
\hline $\mathrm{C} 3-\mathrm{H} 3 \mathrm{~B}$ & 0.9800 & C9- $\mathrm{H} 9 \mathrm{C}$ & 0.9800 \\
\hline $\mathrm{C} 3-\mathrm{H} 3 \mathrm{C}$ & 0.9800 & $\mathrm{C} 11-\mathrm{H} 11 \mathrm{C}$ & 0.9900 \\
\hline $\mathrm{O} 4-\mathrm{C} 5$ & $1.2581(19)$ & $\mathrm{C} 11-\mathrm{H} 11 \mathrm{D}$ & 0.9900 \\
\hline $\mathrm{C} 4-\mathrm{H} 4 \mathrm{~A}$ & 0.9800 & $\mathrm{C} 11-\mathrm{C} 10$ & $1.526(10)$ \\
\hline
\end{tabular}




\begin{tabular}{|c|c|c|c|}
\hline $\mathrm{C} 4-\mathrm{H} 4 \mathrm{~B}$ & 0.9800 & $\mathrm{C} 12-\mathrm{H} 12 \mathrm{C}$ & 0.9900 \\
\hline $\mathrm{C} 4-\mathrm{H} 4 \mathrm{C}$ & 0.9800 & $\mathrm{C} 12-\mathrm{H} 12 \mathrm{D}$ & 0.9900 \\
\hline $\mathrm{O} 5 \mathrm{~A}-\mathrm{H} 5 \mathrm{~A}$ & 0.8400 & $\mathrm{C} 12-\mathrm{C} 13$ & $1.471(18)$ \\
\hline $\mathrm{O} 5 \mathrm{~A}-\mathrm{C} 11 \mathrm{~A}$ & $1.27(2)$ & $\mathrm{C} 13-\mathrm{H} 13 \mathrm{C}$ & 0.9900 \\
\hline $\mathrm{C} 5-\mathrm{C} 6$ & $1.524(2)$ & $\mathrm{C} 13-\mathrm{H} 13 \mathrm{D}$ & 0.9900 \\
\hline O6A-H6A & 0.8400 & $\mathrm{C} 10-\mathrm{H} 10 \mathrm{C}$ & 0.9900 \\
\hline $\mathrm{O} 6 \mathrm{~A}-\mathrm{C} 13 \mathrm{~A}$ & $1.46(6)$ & $\mathrm{C} 10-\mathrm{H} 10 \mathrm{D}$ & 0.9900 \\
\hline $\mathrm{O} 1-\mathrm{Sr} 1-\mathrm{O} 1^{\mathrm{i}}$ & $71.51(4)$ & $\mathrm{H} 4 \mathrm{~A}-\mathrm{C} 4-\mathrm{H} 4 \mathrm{C}$ & 109.5 \\
\hline $\mathrm{O} 1-\mathrm{Sr} 1-\mathrm{N} 1$ & $80.86(4)$ & $\mathrm{H} 4 \mathrm{~B}-\mathrm{C} 4-\mathrm{H} 4 \mathrm{C}$ & 109.5 \\
\hline $\mathrm{O} 1 \mathrm{i}-\mathrm{Sr} 1-\mathrm{N} 1$ & $127.74(4)$ & $\mathrm{Sr} 1-\mathrm{O} 5 \mathrm{~A}-\mathrm{H} 5 \mathrm{~A}$ & 102.1 \\
\hline $\mathrm{O} 1-\mathrm{Sr} 1-\mathrm{O} 2^{\mathrm{i}}$ & $119.26(3)$ & $\mathrm{C} 11 \mathrm{~A}-\mathrm{O} 5 \mathrm{~A}-\mathrm{Sr} 1$ & $128.5(14)$ \\
\hline $\mathrm{O} 1-\mathrm{Sr} 1-\mathrm{O} 3$ & $163.08(4)$ & $\mathrm{C} 11 \mathrm{~A}-\mathrm{O} 5 \mathrm{~A}-\mathrm{H} 5 \mathrm{~A}$ & 109.5 \\
\hline $\mathrm{O} 1-\mathrm{Sr} 1-\mathrm{O} 3^{\mathrm{ii}}$ & $120.63(3)$ & $\mathrm{O} 3-\mathrm{C} 5-\mathrm{Sr}^{\mathrm{ii}}$ & $64.42(8)$ \\
\hline $\mathrm{O} 1-\mathrm{Sr} 1-\mathrm{O} 4^{\mathrm{ii}}$ & $72.27(3)$ & $\mathrm{O} 3-\mathrm{C} 5-\mathrm{O} 4$ & $122.22(14)$ \\
\hline $\mathrm{O} 1-\mathrm{Sr} 1-\mathrm{O} 5 \mathrm{~A}$ & $98.5(4)$ & $\mathrm{O} 3-\mathrm{C} 5-\mathrm{C} 6$ & $119.20(14)$ \\
\hline $\mathrm{O} 1^{\mathrm{i}}-\mathrm{Sr} 1-\mathrm{C} 5^{\mathrm{ii}}$ & $97.57(4)$ & $\mathrm{O} 4-\mathrm{C} 5-\mathrm{Sr}^{\mathrm{ii}}$ & $60.41(8)$ \\
\hline $\mathrm{O} 1-\mathrm{Sr} 1-\mathrm{C} 5^{\mathrm{ii}}$ & $96.16(4)$ & $\mathrm{O} 4-\mathrm{C} 5-\mathrm{C} 6$ & $118.51(13)$ \\
\hline $\mathrm{O} 1-\mathrm{Sr} 1-\mathrm{O} 6 \mathrm{~A}$ & $75.4(11)$ & $\mathrm{C} 6-\mathrm{C} 5-\mathrm{Sr} 1^{\mathrm{ii}}$ & $160.72(11)$ \\
\hline $\mathrm{O} 1-\mathrm{Sr} 1-\mathrm{O} 6$ & $77.3(6)$ & $\mathrm{Sr} 1-\mathrm{O} 6 \mathrm{~A}-\mathrm{H} 6 \mathrm{~A}$ & 120.7 \\
\hline $\mathrm{O} 1-\mathrm{Sr} 1-\mathrm{O} 5$ & $94.8(2)$ & $\mathrm{C} 13 \mathrm{~A}-\mathrm{O} 6 \mathrm{~A}-\mathrm{Sr} 1$ & $121(3)$ \\
\hline $\mathrm{N} 1-\mathrm{Sr} 1-\mathrm{C}^{\mathrm{ii}}$ & $129.42(4)$ & $\mathrm{C} 13 \mathrm{~A}-\mathrm{O} 6 \mathrm{~A}-\mathrm{H} 6 \mathrm{~A}$ & 109.5 \\
\hline $\mathrm{O} 2^{\mathrm{i}}-\mathrm{Sr} 1-\mathrm{O} 1^{\mathrm{i}}$ & $48.14(3)$ & $\mathrm{C} 5-\mathrm{C} 6-\mathrm{H} 6$ & 108.6 \\
\hline $\mathrm{O} 2^{\mathrm{i}}-\mathrm{Sr} 1-\mathrm{N} 1$ & $128.06(4)$ & $\mathrm{C} 5-\mathrm{C} 6-\mathrm{C} 7$ & $110.42(15)$ \\
\hline $\mathrm{O} 2^{\mathrm{i}}-\mathrm{Sr} 1-\mathrm{O} 3^{\mathrm{ii}}$ & $83.06(4)$ & $\mathrm{C} 7-\mathrm{C} 6-\mathrm{H} 6$ & 108.6 \\
\hline $\mathrm{O} 2^{\mathrm{i}}-\mathrm{Sr} 1-\mathrm{O} 4^{\mathrm{ii}}$ & $104.22(4)$ & $\mathrm{C} 8-\mathrm{C} 6-\mathrm{C} 5$ & $109.65(15)$ \\
\hline $\mathrm{O} 2^{\mathrm{i}}-\mathrm{Sr} 1-\mathrm{C} 5^{\mathrm{ii}}$ & $97.57(4)$ & $\mathrm{C} 8-\mathrm{C} 6-\mathrm{H} 6$ & 108.6 \\
\hline $\mathrm{O} 2^{\mathrm{i}}-\mathrm{Sr} 1-\mathrm{O} 6 \mathrm{~A}$ & $75.6(12)$ & $\mathrm{C} 8-\mathrm{C} 6-\mathrm{C} 7$ & $110.99(16)$ \\
\hline $\mathrm{O} 2^{\mathrm{i}}-\mathrm{Sr} 1-\mathrm{O} 6$ & $76.6(7)$ & $\mathrm{C} 6-\mathrm{C} 7-\mathrm{H} 7 \mathrm{~A}$ & 109.5 \\
\hline $\mathrm{O} 2^{\mathrm{i}}-\mathrm{Sr} 1-\mathrm{O} 5$ & $144.7(2)$ & $\mathrm{C} 6-\mathrm{C} 7-\mathrm{H} 7 \mathrm{~B}$ & 109.5 \\
\hline $\mathrm{O} 3-\mathrm{Sr} 1-\mathrm{O} 1^{\mathrm{i}}$ & $120.02(3)$ & $\mathrm{C} 6-\mathrm{C} 7-\mathrm{H} 7 \mathrm{C}$ & 109.5 \\
\hline $\mathrm{O} 3^{\mathrm{ii}}-\mathrm{Sr} 1-\mathrm{O} 1^{\mathrm{i}}$ & $102.22(3)$ & $\mathrm{H} 7 \mathrm{~A}-\mathrm{C} 7-\mathrm{H} 7 \mathrm{~B}$ & 109.5 \\
\hline $\mathrm{O} 3-\mathrm{Sr} 1-\mathrm{N} 1$ & $82.22(4)$ & $\mathrm{H} 7 \mathrm{~A}-\mathrm{C} 7-\mathrm{H} 7 \mathrm{C}$ & 109.5 \\
\hline $\mathrm{O} 3^{\mathrm{ii}}-\mathrm{Sr} 1-\mathrm{N} 1$ & $130.04(4)$ & $\mathrm{H} 7 \mathrm{~B}-\mathrm{C} 7-\mathrm{H} 7 \mathrm{C}$ & 109.5 \\
\hline $\mathrm{O} 3-\mathrm{Sr} 1-\mathrm{O} 2^{\mathrm{i}}$ & $72.11(3)$ & $\mathrm{C} 6-\mathrm{C} 8-\mathrm{H} 8 \mathrm{~A}$ & 109.5 \\
\hline $\mathrm{O} 3-\mathrm{Sr} 1-\mathrm{O} 3^{\mathrm{ii}}$ & $71.14(4)$ & $\mathrm{C} 6-\mathrm{C} 8-\mathrm{H} 8 \mathrm{~B}$ & 109.5 \\
\hline $\mathrm{O} 3-\mathrm{Sr} 1-\mathrm{O} 4^{\mathrm{ii}}$ & $118.97(3)$ & $\mathrm{C} 6-\mathrm{C} 8-\mathrm{H} 8 \mathrm{C}$ & 109.5 \\
\hline $\mathrm{O} 3-\mathrm{Sr} 1-\mathrm{O} 5 \mathrm{~A}$ & $72.5(4)$ & $\mathrm{H} 8 \mathrm{~A}-\mathrm{C} 8-\mathrm{H} 8 \mathrm{~B}$ & 109.5 \\
\hline $\mathrm{O} 3-\mathrm{Sr} 1-\mathrm{C} 5^{\mathrm{ii}}$ & $94.46(4)$ & $\mathrm{H} 8 \mathrm{~A}-\mathrm{C} 8-\mathrm{H} 8 \mathrm{C}$ & 109.5 \\
\hline $\mathrm{O} 3^{\mathrm{ii}}-\mathrm{Sr} 1-\mathrm{C}^{\mathrm{ii}}$ & $24.58(4)$ & $\mathrm{H} 8 \mathrm{~B}-\mathrm{C} 8-\mathrm{H} 8 \mathrm{C}$ & 109.5 \\
\hline $\mathrm{O} 3-\mathrm{Sr} 1-\mathrm{O} 6 \mathrm{~A}$ & $97.1(10)$ & N1-C9A-H9AA & 109.5 \\
\hline $\mathrm{O} 3-\mathrm{Sr} 1-\mathrm{O} 6$ & $94.5(6)$ & $\mathrm{N} 1-\mathrm{C} 9 \mathrm{~A}-\mathrm{H} 9 \mathrm{AB}$ & 109.5 \\
\hline $\mathrm{O} 3^{\mathrm{ii}}-\mathrm{Sr} 1-\mathrm{O} 5$ & $70.7(2)$ & $\mathrm{N} 1-\mathrm{C} 9 \mathrm{~A}-\mathrm{H} 9 \mathrm{AC}$ & 109.5 \\
\hline $\mathrm{O} 3-\mathrm{Sr} 1-\mathrm{O} 5$ & $77.2(2)$ & H9AA-C9A-H9AB & 109.5 \\
\hline $\mathrm{O} 4^{\mathrm{ii}-}-\mathrm{Sr} 1-\mathrm{O} 1^{\mathrm{i}}$ & $84.80(3)$ & H9AA-C9A-H9AC & 109.5 \\
\hline $\mathrm{O} 4^{\mathrm{ii}}-\mathrm{Sr} 1-\mathrm{N} 1$ & $127.72(4)$ & $\mathrm{H} 9 \mathrm{AB}-\mathrm{C} 9 \mathrm{~A}-\mathrm{H} 9 \mathrm{AC}$ & 109.5 \\
\hline $\mathrm{O} 4^{\mathrm{ii}}-\mathrm{Sr} 1-\mathrm{O} 3^{\mathrm{ii}}$ & $48.46(3)$ & $\mathrm{N} 1-\mathrm{C} 10 \mathrm{~A}-\mathrm{H} 10 \mathrm{~A}$ & 108.4 \\
\hline $\mathrm{O} 4^{\mathrm{ii}-}-\mathrm{Sr} 1-\mathrm{C} 5^{\mathrm{ii}}$ & $24.52(4)$ & $\mathrm{N} 1-\mathrm{C} 10 \mathrm{~A}-\mathrm{H} 10 \mathrm{~B}$ & 108.4 \\
\hline
\end{tabular}




\begin{tabular}{|c|c|}
\hline $\mathrm{O} 4^{\mathrm{ii}}-\mathrm{Sr} 1-\mathrm{O} 6 \mathrm{~A}$ & $142.4(9)$ \\
\hline $\mathrm{O} 4^{\mathrm{ii}}-\mathrm{Sr} 1-\mathrm{O} 6$ & $145.4(6)$ \\
\hline $\mathrm{O} 4^{\mathrm{ii}}-\mathrm{Sr} 1-\mathrm{O} 5$ & $75.82(16)$ \\
\hline $\mathrm{O} 5 \mathrm{~A}-\mathrm{Sr} 1-\mathrm{N} 1$ & $59.3(4)$ \\
\hline $\mathrm{O} 5 \mathrm{~A}-\mathrm{Sr} 1-\mathrm{C}^{\mathrm{ii}}$ & $71.5(4)$ \\
\hline $\mathrm{O} 5 \mathrm{~A}-\mathrm{Sr} 1-\mathrm{O} 6 \mathrm{~A}$ & $122.7(11)$ \\
\hline $\mathrm{O} 6 \mathrm{~A}-\mathrm{Sr} 1-\mathrm{N} 1$ & $63.5(10)$ \\
\hline $\mathrm{O} 6 \mathrm{~A}-\mathrm{Sr} 1-\mathrm{C} 5^{\mathrm{ii}}$ & $164.0(9)$ \\
\hline $\mathrm{O} 6-\mathrm{Sr} 1-\mathrm{O} 1^{\mathrm{i}}$ & $70.0(6)$ \\
\hline $\mathrm{O} 6-\mathrm{Sr} 1-\mathrm{N} 1$ & $61.0(6)$ \\
\hline $\mathrm{O} 6-\mathrm{Sr} 1-\mathrm{O} 3^{\mathrm{ii}}$ & $158.1(7)$ \\
\hline $\mathrm{O} 6-\mathrm{Sr} 1-\mathrm{C} 5^{\mathrm{ii}}$ & $167.2(5)$ \\
\hline $\mathrm{O} 6-\mathrm{Sr} 1-\mathrm{O} 5$ & $123.5(7)$ \\
\hline $\mathrm{O} 5-\mathrm{Sr} 1-\mathrm{O} 1^{\mathrm{i}}$ & $159.08(16)$ \\
\hline $\mathrm{O} 5-\mathrm{Sr} 1-\mathrm{N} 1$ & $62.5(2)$ \\
\hline $\mathrm{O} 5-\mathrm{Sr} 1-\mathrm{C} 5^{\mathrm{ii}}$ & $67.5(2)$ \\
\hline $\mathrm{Sr} 1-\mathrm{O} 1-\mathrm{Sr}^{\mathrm{i}}$ & $108.50(4)$ \\
\hline $\mathrm{Sr} 1-\mathrm{O} 1-\mathrm{Sr} 1^{\mathrm{ii}}$ & $108.84(5)$ \\
\hline $\mathrm{C} 1-\mathrm{O} 1-\mathrm{Sr} 1$ & $154.61(10)$ \\
\hline $\mathrm{C} 1-\mathrm{O} 1-\mathrm{Sr} 1^{\mathrm{i}}$ & $90.10(9)$ \\
\hline $\mathrm{C} 9 \mathrm{~A}-\mathrm{N} 1-\mathrm{Sr} 1$ & $110.3(2)$ \\
\hline $\mathrm{C} 10 \mathrm{~A}-\mathrm{N} 1-\mathrm{Sr} 1$ & $106.8(2)$ \\
\hline $\mathrm{C} 10 \mathrm{~A}-\mathrm{N} 1-\mathrm{C} 9 \mathrm{~A}$ & $107.4(3)$ \\
\hline $\mathrm{C} 12 \mathrm{~A}-\mathrm{N} 1-\mathrm{Sr} 1$ & $106.99(19)$ \\
\hline $\mathrm{C} 12 \mathrm{~A}-\mathrm{N} 1-\mathrm{C} 9 \mathrm{~A}$ & $108.1(3)$ \\
\hline $\mathrm{C} 12 \mathrm{~A}-\mathrm{N} 1-\mathrm{C} 10 \mathrm{~A}$ & $117.2(3)$ \\
\hline $\mathrm{C} 9-\mathrm{N} 1-\mathrm{Sr} 1$ & $113.27(13)$ \\
\hline $\mathrm{C} 12-\mathrm{N} 1-\mathrm{Sr} 1$ & $107.93(14)$ \\
\hline $\mathrm{C} 12-\mathrm{N} 1-\mathrm{C} 9$ & $107.2(2)$ \\
\hline $\mathrm{C} 10-\mathrm{N} 1-\mathrm{Sr} 1$ & $106.71(13)$ \\
\hline $\mathrm{C} 10-\mathrm{N} 1-\mathrm{C} 9$ & $108.39(19)$ \\
\hline $\mathrm{C} 10-\mathrm{N} 1-\mathrm{C} 12$ & $113.45(19)$ \\
\hline $\mathrm{O} 1-\mathrm{C} 1-\mathrm{Sr}^{\mathrm{i}}$ & $65.45(8)$ \\
\hline $\mathrm{O} 1-\mathrm{C} 1-\mathrm{O} 2$ & $122.18(14)$ \\
\hline $\mathrm{O} 1-\mathrm{C} 1-\mathrm{C} 2$ & $119.64(13)$ \\
\hline $\mathrm{O} 2-\mathrm{C} 1-\mathrm{Sr} 1^{\mathrm{i}}$ & $59.55(8)$ \\
\hline $\mathrm{O} 2-\mathrm{C} 1-\mathrm{C} 2$ & $118.10(13)$ \\
\hline $\mathrm{C} 2-\mathrm{C} 1-\mathrm{Sr} 1^{\mathrm{i}}$ & $159.64(11)$ \\
\hline $\mathrm{C} 1-\mathrm{O} 2-\mathrm{Sr}^{\mathrm{i}}$ & $96.07(9)$ \\
\hline $\mathrm{C} 1-\mathrm{C} 2-\mathrm{H} 2$ & 108.8 \\
\hline $\mathrm{C} 3-\mathrm{C} 2-\mathrm{C} 1$ & $109.41(14)$ \\
\hline $\mathrm{C} 3-\mathrm{C} 2-\mathrm{H} 2$ & 108.8 \\
\hline $\mathrm{C} 4-\mathrm{C} 2-\mathrm{C} 1$ & $109.84(15)$ \\
\hline $\mathrm{C} 4-\mathrm{C} 2-\mathrm{H} 2$ & 108.8 \\
\hline $\mathrm{C} 4-\mathrm{C} 2-\mathrm{C} 3$ & $111.17(17)$ \\
\hline $\mathrm{Sr} 1-\mathrm{O} 3-\mathrm{Sr} 1^{\mathrm{ii}}$ & $108.86(4)$ \\
\hline $\mathrm{C} 5-\mathrm{O} 3-\mathrm{Sr} 1^{\mathrm{ii}}$ & $91.00(9)$ \\
\hline $\mathrm{C} 5-\mathrm{O} 3-\mathrm{Sr} 1$ & $152.58(10)$ \\
\hline
\end{tabular}

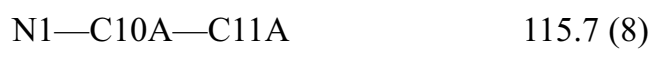

$115.7(8)$

107.4

108.4

108.4

112.5 (14)

109.1

109.1

109.1

109.1

107.8

108.5

108.5

$115.2(12)$

107.5

108.5

108.5

$110(3)$

109.7

109.7

109.7

109.7

108.2

97.5

$122(2)$

109.5

98.5

117.7 (5)

109.5

109.5

109.5

109.5

109.5

109.5

109.5

109.1

109.1

107.9

112.4 (6)

109.1

109.1

108.8

108.8

$113.6(8)$

107.7

108.8

108.8

106.9 (17)

110.3 


\begin{tabular}{|c|c|c|c|}
\hline $\mathrm{C} 2-\mathrm{C} 3-\mathrm{H} 3 \mathrm{~A}$ & 109.5 & $\mathrm{O} 6-\mathrm{C} 13-\mathrm{H} 13 \mathrm{D}$ & 110.3 \\
\hline $\mathrm{C} 2-\mathrm{C} 3-\mathrm{H} 3 \mathrm{~B}$ & 109.5 & $\mathrm{C} 12-\mathrm{C} 13-\mathrm{H} 13 \mathrm{C}$ & 110.3 \\
\hline $\mathrm{C} 2-\mathrm{C} 3-\mathrm{H} 3 \mathrm{C}$ & 109.5 & $\mathrm{C} 12-\mathrm{C} 13-\mathrm{H} 13 \mathrm{D}$ & 110.3 \\
\hline $\mathrm{H} 3 \mathrm{~A}-\mathrm{C} 3-\mathrm{H} 3 \mathrm{~B}$ & 109.5 & $\mathrm{H} 13 \mathrm{C}-\mathrm{C} 13-\mathrm{H} 13 \mathrm{D}$ & 108.6 \\
\hline $\mathrm{H} 3 \mathrm{~A}-\mathrm{C} 3-\mathrm{H} 3 \mathrm{C}$ & 109.5 & $\mathrm{~N} 1-\mathrm{C} 10-\mathrm{C} 11$ & $115.8(3)$ \\
\hline $\mathrm{H} 3 \mathrm{~B}-\mathrm{C} 3-\mathrm{H} 3 \mathrm{C}$ & 109.5 & $\mathrm{~N} 1-\mathrm{C} 10-\mathrm{H} 10 \mathrm{C}$ & 108.3 \\
\hline $\mathrm{C} 5-\mathrm{O} 4-\mathrm{Sr}^{\mathrm{ii}}$ & $95.07(9)$ & $\mathrm{N} 1-\mathrm{C} 10-\mathrm{H} 10 \mathrm{D}$ & 108.3 \\
\hline $\mathrm{C} 2-\mathrm{C} 4-\mathrm{H} 4 \mathrm{~A}$ & 109.5 & $\mathrm{C} 11-\mathrm{C} 10-\mathrm{H} 10 \mathrm{C}$ & 108.3 \\
\hline $\mathrm{C} 2-\mathrm{C} 4-\mathrm{H} 4 \mathrm{~B}$ & 109.5 & $\mathrm{C} 11-\mathrm{C} 10-\mathrm{H} 10 \mathrm{D}$ & 108.3 \\
\hline $\mathrm{C} 2-\mathrm{C} 4-\mathrm{H} 4 \mathrm{C}$ & 109.5 & $\mathrm{H} 10 \mathrm{C}-\mathrm{C} 10-\mathrm{H} 10 \mathrm{D}$ & 107.4 \\
\hline $\mathrm{H} 4 \mathrm{~A}-\mathrm{C} 4-\mathrm{H} 4 \mathrm{~B}$ & 109.5 & & \\
\hline $\mathrm{Sr} 1-\mathrm{O} 1-\mathrm{C} 1-\mathrm{Sr}^{\mathrm{i}}$ & $138.0(2)$ & $\mathrm{Sr} 1-\mathrm{O} 5-\mathrm{C} 11-\mathrm{C} 10$ & $1.0(9)$ \\
\hline $\mathrm{Sr} 1-\mathrm{O} 1-\mathrm{C} 1-\mathrm{O} 2$ & $18.98(15)$ & $\mathrm{O} 1-\mathrm{C} 1-\mathrm{O} 2-\mathrm{Sr} 1^{\mathrm{i}}$ & $-20.07(16)$ \\
\hline $\mathrm{Sr} 1-\mathrm{O} 1-\mathrm{C} 1-\mathrm{O} 2$ & $157.02(16)$ & $\mathrm{O} 1-\mathrm{C} 1-\mathrm{C} 2-\mathrm{C} 3$ & $113.07(18)$ \\
\hline $\mathrm{Sr} 1-\mathrm{O} 1-\mathrm{C} 1-\mathrm{C} 2$ & $-157.82(13)$ & $\mathrm{O} 1-\mathrm{C} 1-\mathrm{C} 2-\mathrm{C} 4$ & $-124.64(17)$ \\
\hline $\mathrm{Sr} 1-\mathrm{O} 1-\mathrm{C} 1-\mathrm{C} 2$ & $-19.8(3)$ & $\mathrm{N} 1-\mathrm{C} 10 \mathrm{~A}-\mathrm{C} 11 \mathrm{~A}-\mathrm{O} 5 \mathrm{~A}$ & $42.3(14)$ \\
\hline $\mathrm{Sr} 1-\mathrm{N} 1-\mathrm{C} 10 \mathrm{~A}-\mathrm{C} 11 \mathrm{~A}$ & $-46.2(7)$ & $\mathrm{N} 1-\mathrm{C} 12 \mathrm{~A}-\mathrm{C} 13 \mathrm{~A}-\mathrm{O} 6 \mathrm{~A}$ & $-49(3)$ \\
\hline $\mathrm{Sr} 1-\mathrm{N} 1-\mathrm{C} 12 \mathrm{~A}-\mathrm{C} 13 \mathrm{~A}$ & $54.3(13)$ & $\mathrm{N} 1-\mathrm{C} 12-\mathrm{C} 13-\mathrm{O} 6$ & $61.3(18)$ \\
\hline $\mathrm{Sr} 1-\mathrm{N} 1-\mathrm{C} 12-\mathrm{C} 13$ & $-50.4(9)$ & $\mathrm{O} 2-\mathrm{C} 1-\mathrm{C} 2-\mathrm{C} 3$ & $-63.9(2)$ \\
\hline $\mathrm{Sr} 1-\mathrm{N} 1-\mathrm{C} 10-\mathrm{C} 11$ & $57.0(5)$ & $\mathrm{O} 2-\mathrm{C} 1-\mathrm{C} 2-\mathrm{C} 4$ & $58.4(2)$ \\
\hline $\mathrm{Sr} 1-\mathrm{C} 1-\mathrm{C} 2-\mathrm{C} 3$ & $13.9(4)$ & $\mathrm{C} 2-\mathrm{C} 1-\mathrm{O} 2-\mathrm{Sr}{ }^{\mathrm{i}}$ & $156.78(12)$ \\
\hline $\mathrm{Sr} 1-\mathrm{C} 1-\mathrm{C} 2-\mathrm{C} 4$ & $136.2(3)$ & $\mathrm{O} 3-\mathrm{C} 5-\mathrm{C} 6-\mathrm{C} 7$ & $-123.02(18)$ \\
\hline $\mathrm{Sr} 1-\mathrm{O} 3-\mathrm{C} 5-\mathrm{Sr}^{\mathrm{ii}}$ & $137.4(2)$ & $\mathrm{O} 3-\mathrm{C} 5-\mathrm{C} 6-\mathrm{C} 8$ & $114.37(17)$ \\
\hline $\mathrm{Sr}^{\mathrm{ii}}-\mathrm{O} 3-\mathrm{C} 5-\mathrm{O} 4$ & $18.43(16)$ & $\mathrm{O} 4-\mathrm{C} 5-\mathrm{C} 6-\mathrm{C} 7$ & $59.8(2)$ \\
\hline $\mathrm{Sr} 1-\mathrm{O} 3-\mathrm{C} 5-\mathrm{O} 4$ & $155.79(15)$ & $\mathrm{O} 4-\mathrm{C} 5-\mathrm{C} 6-\mathrm{C} 8$ & $-62.8(2)$ \\
\hline $\mathrm{Sr}^{\mathrm{ii}}-\mathrm{O} 3-\mathrm{C} 5-\mathrm{C} 6$ & $-158.64(13)$ & $\mathrm{C} 9 \mathrm{~A}-\mathrm{N} 1-\mathrm{C} 10 \mathrm{~A}-\mathrm{C} 11 \mathrm{~A}$ & $72.0(7)$ \\
\hline $\mathrm{Sr} 1-\mathrm{O} 3-\mathrm{C} 5-\mathrm{C} 6$ & $-21.3(3)$ & $\mathrm{C} 9 \mathrm{~A}-\mathrm{N} 1-\mathrm{C} 12 \mathrm{~A}-\mathrm{C} 13 \mathrm{~A}$ & $-64.4(13)$ \\
\hline $\mathrm{Sr}^{\mathrm{ii}}-\mathrm{O} 4-\mathrm{C} 5-\mathrm{O} 3$ & $-19.14(16)$ & $\mathrm{C} 10 \mathrm{~A}-\mathrm{N} 1-\mathrm{C} 12 \mathrm{~A}-\mathrm{C} 13 \mathrm{~A}$ & $174.1(13)$ \\
\hline $\mathrm{Sr}^{\mathrm{ii}}-\mathrm{O} 4-\mathrm{C} 5-\mathrm{C} 6$ & $157.94(13)$ & $\mathrm{C} 12 \mathrm{~A}-\mathrm{N} 1-\mathrm{C} 10 \mathrm{~A}-\mathrm{C} 11 \mathrm{~A}$ & $-166.1(6)$ \\
\hline $\mathrm{Sr} 1-\mathrm{O} 5 \mathrm{~A}-\mathrm{C} 11 \mathrm{~A}-\mathrm{C} 10 \mathrm{~A}$ & $-12.8(19)$ & $\mathrm{O} 5-\mathrm{C} 11-\mathrm{C} 10-\mathrm{N} 1$ & $-41.3(8)$ \\
\hline $\mathrm{Sr} 1^{\mathrm{ii}}-\mathrm{C} 5-\mathrm{C} 6-\mathrm{C} 7$ & $141.2(3)$ & $\mathrm{C} 9-\mathrm{N} 1-\mathrm{C} 12-\mathrm{C} 13$ & $72.0(9)$ \\
\hline $\mathrm{Sr} 1^{\mathrm{ii}}-\mathrm{C} 5-\mathrm{C} 6-\mathrm{C} 8$ & $18.6(4)$ & $\mathrm{C} 9-\mathrm{N} 1-\mathrm{C} 10-\mathrm{C} 11$ & $-65.3(5)$ \\
\hline $\mathrm{Sr} 1-\mathrm{O} 6 \mathrm{~A}-\mathrm{C} 13 \mathrm{~A}-\mathrm{C} 12 \mathrm{~A}$ & $15(4)$ & $\mathrm{C} 12-\mathrm{N} 1-\mathrm{C} 10-\mathrm{C} 11$ & $175.7(5)$ \\
\hline $\mathrm{Sr} 1-\mathrm{O} 6-\mathrm{C} 13-\mathrm{C} 12$ & $-41(2)$ & $\mathrm{C} 10-\mathrm{N} 1-\mathrm{C} 12-\mathrm{C} 13$ & $-168.4(9)$ \\
\hline
\end{tabular}

Symmetry codes: (i) $-x+2,-y+1,-z+1$; (ii) $-x+1,-y+1,-z+1$.

Hydrogen-bond geometry $\left(\AA,{ }^{\circ}\right)$

\begin{tabular}{lllll}
\hline$D-\mathrm{H} \cdots A$ & $D-\mathrm{H}$ & $\mathrm{H} \cdots A$ & $D \cdots A$ & $D-\mathrm{H}^{\cdots} A$ \\
\hline $\mathrm{O} 6 A-\mathrm{H} 6 A \cdots \mathrm{O} 4^{\text {iii }}$ & 0.84 & 1.83 & $2.61(5)$ & 153 \\
$\mathrm{O} 6-\mathrm{H} 6 B^{\mathrm{ii}} \mathrm{O} 4^{\text {iii }}$ & 0.84 & 1.95 & $2.75(3)$ & 160 \\
$\mathrm{O} 5-\mathrm{H} 5 \cdots \mathrm{O} 2^{\text {iv }}$ & 0.84 & 1.87 & $2.680(12)$ & 163 \\
\hline
\end{tabular}

Symmetry codes: (iii) $x+1, y, z$; (iv) $x-1, y, z$. 
Selected bond lengths ( $(\AA)$ and angles $\left({ }^{\circ}\right)$.

\begin{tabular}{llll}
\hline $\mathrm{Sr} 1-\mathrm{O} 1^{\mathrm{i}}$ & $2.7568(13)$ & $\mathrm{Sr} 1-\mathrm{O} 6$ & $2.655(4)$ \\
$\mathrm{S} 1-\mathrm{O}^{\mathrm{ii}}$ & $2.7251(14)$ & $\mathrm{S} 1-\mathrm{N} 1$ & $2.8516(16)$ \\
$\mathrm{Sr} 1-\mathrm{O} 1$ & $2.5370(12)$ & $\mathrm{Sr} 1 \cdots \mathrm{Sr} 1^{\mathrm{i}}$ & $4.2981(3)$ \\
$\mathrm{Sr} 1-\mathrm{O} 3$ & $2.5441(13)$ & $\mathrm{Sr} 1 \cdots \mathrm{Sr} 1^{\mathrm{ii}}$ & $4.2868(3)$ \\
$\mathrm{Sr} 1-\mathrm{O} 2^{\mathrm{i}}$ & $2.6273(14)$ & $\mathrm{Sr} 1-\mathrm{O} 1-\mathrm{Sr} 1^{\mathrm{i}}$ & $108.50(4)$ \\
$\mathrm{Sr} 1-\mathrm{O} 4^{\mathrm{ii}}$ & $2.6367(14)$ & $\mathrm{Sr} 1-\mathrm{O} 1-\mathrm{Sr} 1^{\mathrm{ii}}$ & $108.84(5)$ \\
$\mathrm{Sr} 1-\mathrm{O} 5$ & $2.678(4)$ & & \\
\hline
\end{tabular}

Symmetry codes: (i) $2-\mathrm{x}, 1-\mathrm{y}, 1-\mathrm{z}$; (ii) $1-\mathrm{x}, 1-\mathrm{y}, 1-\mathrm{z}$. 